\title{
Energy Scattering for Schrödinger Equation with Exponential Nonlinearity in Two Dimensions
}

\author{
Shuxia Wang \\ School of Mathematical Sciences, Peking University, Beijing 100871, China \\ Correspondence should be addressed to Shuxia Wang; wangshuxia@pku.edu.cn
}

Received 9 January 2013; Accepted 24 February 2013

Academic Editor: Baoxiang Wang

Copyright (C) 2013 Shuxia Wang. This is an open access article distributed under the Creative Commons Attribution License, which permits unrestricted use, distribution, and reproduction in any medium, provided the original work is properly cited.

When the spatial dimensions $n=2$, the initial data $u_{0} \in H^{1}$, and the Hamiltonian $H\left(u_{0}\right) \leq 1$, we prove that the scattering operator is well defined in the whole energy space $H^{1}\left(\mathbb{R}^{2}\right)$ for nonlinear Schrödinger equation with exponential nonlinearity $\left(e^{\lambda|u|^{2}}-1\right) u$, where $0<\lambda<4 \pi$.

\section{Introduction}

We consider the Cauchy problem for the following nonlinear Schrödinger equation:

$$
\begin{gathered}
i u_{t}+\Delta u=f(u), \\
f(u):=\left(e^{\lambda|u|^{2}}-1\right) u,
\end{gathered}
$$

in two spatial dimensions with initial data $u_{0} \in H^{1}$ and $0<\lambda<4 \pi$. Solutions of the above problem satisfy the conservation of mass and Hamiltonian:

$$
\begin{gathered}
M(u ; t):=\int_{\mathbb{R}^{2}}|u|^{2} d x=M\left(u_{0}\right), \\
H(u ; t):=\int_{\mathbb{R}^{2}}\left(|\nabla u|^{2}+F(u)\right) d x=H\left(u_{0}\right),
\end{gathered}
$$

where

$$
F(u)=\frac{1}{\lambda}\left(e^{\lambda|u|^{2}}-\lambda|u|^{2}-1\right) .
$$

Nakamura and Ozawa [1] showed the existence and uniqueness of the scattering operator of (1) with (2). Then, Wang [2] proved the smoothness of this scattering operator. However, both of these results are based on the assumption of small initial data $u_{0}$. In this paper, we remove this assumption and show that for arbitrary initial data $u_{0} \in H^{1}\left(\mathbb{R}^{2}\right)$ and $H\left(u_{0}\right) \leq 1$, the scattering operator is always well defined.
Wang et al. [3] proved the energy scattering theory of (1) with $f(u)=\left(e^{\lambda|u|^{2}}-1-\lambda|u|^{2}-\left(\lambda^{2} / 2\right)|u|^{4}\right) u$, where $\lambda \in \mathbb{R}$ and the spatial dimension $n=1$. Ibrahim et al. [4] showed the existence and asymptotic completeness of the wave operators for (1) with $f(u)=\left(e^{\lambda|u|^{2}}-1-\lambda|u|^{2}\right) u$ when the spatial dimensions $n=2, \lambda=4 \pi$, and $H\left(u_{0}\right) \leq 1$. Under the same assumptions as [4], Colliander et al. [5] proved the global well-posedness of (1) with (2).

Theorem 1. Assume that $u_{0} \in H^{1}\left(\mathbb{R}^{2}\right), H\left(u_{0}\right) \leq 1$, and $\lambda=$ $4 \pi$. Then problem (1) with (2) has a unique global solution $u$ in the class $C\left(\mathbb{R}, H^{1}\left(\mathbb{R}^{2}\right)\right)$.

Remark 2. In fact, by the proof in [5], the global wellposedness of (1) with (2) is also true for $0<\lambda \leq 4 \pi$.

In this paper, we further study the scattering of this problem. Note that $f(u)=\left(e^{\lambda|u|^{2}}-1\right) u=\sum_{k=1}^{\infty}\left(\lambda^{k} / k !\right)|u|^{2 k} u$. Nakanishi [6] proved the existence of the scattering operators in the whole energy space $H^{1}\left(\mathbb{R}^{2}\right)$ for (1) with $f(u)=|u|^{p} u$ when $p>2$. Then, Killip et al. [7] and Dodson [8] proved the existence of the scattering operators in $L^{2}\left(\mathbb{R}^{2}\right)$ for (1) with $f(u)=|u|^{2} u$. Inspired by these two works, we use the concentration compactness method, which was introduced by Kenig and Merle in [9], to prove the existence of the scattering operators for (1) with (2). 
For convenience, we write (1) and (2) together; that is,

$$
i u_{t}+\Delta u=f(u):=\left(e^{\lambda|u|^{2}}-1\right) u, \quad u(0, x)=u_{0}
$$

where $u_{0} \in H^{1}\left(\mathbb{R}^{2}\right)$ and $0<\lambda<4 \pi$. Our main result is as follows.

Theorem 3. Assume that the initial data $u_{0} \in H^{1}\left(\mathbb{R}^{2}\right)$, $H\left(u_{0}\right) \leq 1$, and $0<\lambda<4 \pi$. Let $u$ be a global solution of (5). Then

$$
\|u\|_{L_{t, x}^{4}\left(\mathbb{R} \times \mathbb{R}^{2}\right)}<\infty .
$$

In Section 2, Lemma 9 will show us that Theorem 3 implies the following scattering result.

Theorem 4. Assume that the initial data $u_{0} \in H^{1}\left(\mathbb{R}^{2}\right)$, $H\left(u_{0}\right) \leq 1$, and $0<\lambda<4 \pi$. Then the solution of (5) is scattering in the energy space $H^{1}\left(\mathbb{R}^{2}\right)$.

We will prove Theorem 3 by contradiction in Section 5 . In Section 2, we give some nonlinear estimates. In Section 3, we prove the stability of solutions. In Section 4, we give a new profile decomposition for $H^{1}$ sequence which will be used to prove concentration compactness.

Now, we introduce some notations:

$$
\begin{aligned}
G(u) & :=\bar{u} f(u)-F(u)=e^{\lambda|u|^{2}}|u|^{2}-\frac{1}{\lambda}\left(e^{\lambda|u|^{2}}-1\right) \\
& =\sum_{k=1}^{\infty} \frac{k \lambda^{k}|u|^{2 k+2}}{(k+1) !}, \\
E & =E(u ; t):=M(u ; t)+H(u ; t) .
\end{aligned}
$$

We define

$$
\begin{gathered}
\|u\|_{H_{q}^{s}\left(\mathbb{R}^{2}\right)}:=\left\|(I-\triangle)^{s / 2} u\right\|_{L^{q}\left(\mathbb{R}^{2}\right)}, \\
\|u\|_{\dot{H}_{q}^{s}\left(\mathbb{R}^{2}\right)}:=\left\|(-\triangle)^{s / 2} u\right\|_{L^{q}\left(\mathbb{R}^{2}\right)} .
\end{gathered}
$$

For Banach space $X=H_{q}^{s}\left(\mathbb{R}^{2}\right), \dot{H}_{q}^{s}\left(\mathbb{R}^{2}\right)$, or $L^{q}\left(\mathbb{R}^{2}\right)$, we denote

$$
\|u\|_{L^{p}(\mathbb{R} ; X)}:=\left(\int_{\mathbb{R}}\|u(t)\|_{X}^{p} d t\right)^{1 / p} .
$$

When $q=r, L_{t}^{q} L_{x}^{r}$ is abbreviated to $L_{t, x}^{q}$. When $q$ or $r$ is infinity or when the domain $\mathbb{R} \times \mathbb{R}^{2}$ is replaced by $I \times \mathbb{R}^{2}$, we make the usual modifications. Specially, we denote

$$
S(u):=\|u\|_{L_{t, x}^{4}}^{4}\left(\mathbb{R} \times \mathbb{R}^{2}\right) .
$$

For $t_{0} \in \mathbb{R}$, we split $S(u)=S_{\leq t_{0}}(u)+S_{\geq t_{0}}(u)$, where

$$
\begin{aligned}
& S_{\leq t_{0}}(u):=\int_{-\infty}^{t_{0}} \int_{\mathbb{R}^{2}}|u(t, x)|^{4} d x d t, \\
& S_{\geq t_{0}}(u):=\int_{t_{0}}^{+\infty} \int_{\mathbb{R}^{2}}|u(t, x)|^{4} d x d t .
\end{aligned}
$$

For any two Banach spaces $X$ and $Y,\|\cdot\|_{X \cap Y}:=$ $\max \left\{\|\cdot\|_{X},\|\cdot\|_{Y}\right\}$. $C$ denotes positive constant. If $C$ depends upon some parameters, such as $\lambda$, we will indicate this with $C(\lambda)$.

Remark 5. Note that $0<\lambda<4 \pi$ in Theorem 3; we only need to prove the result for $0<\lambda<4(1-4 \varepsilon)^{2} \pi, \varepsilon \in(0,1 / 8)$. Hence, we always suppose that $0<\lambda<4(1-4 \varepsilon)^{2} \pi$ in the context.

Moreover, we always suppose that the initial data $u_{0}$ of (5) satisfies $u_{0} \in H^{1}\left(\mathbb{R}^{2}\right)$ and $H\left(u_{0}\right) \leq 1$.

\section{Nonlinear Estimates}

In order to estimate (2), we need the following Trudinger-type inequality.

Lemma 6 (see [10]). Let $\lambda \in[0,4 \pi)$. Then for all $u \in H^{1}\left(\mathbb{R}^{2}\right)$ satisfying $\|\nabla u\|_{L^{2}\left(\mathbb{R}^{2}\right)} \leq 1$, one has

$$
\int_{\mathbb{R}^{2}}\left(e^{\lambda|u|^{2}}-1\right) d x \leq C(\lambda)\|u\|_{L^{2}\left(\mathbb{R}^{2}\right)}^{2} .
$$

Note that for for all $\alpha \geq 1$,

$$
\left(e^{\lambda|u|^{2}}-1\right)^{\alpha} \leq e^{\lambda \alpha|u|^{2}}-1
$$

By Lemma 6 and Hölder inequality, for $\lambda \in(0,4 \pi)$ and for all $\beta \geq 0$, we have

$$
\begin{aligned}
\int_{\mathbb{R}^{2}}\left(e^{\lambda|u|^{2}}-1\right)|u|^{\beta} d x & \leq\left\|e^{\lambda|u|^{2}}-1\right\|_{L_{x}^{1 /(1-\varepsilon)}\left(\mathbb{R}^{2}\right)}\|u\|_{L_{x}^{\beta / \varepsilon}\left(\mathbb{R}^{2}\right)}^{\beta} \\
& \leq\|u\|_{L^{2}\left(\mathbb{R}^{2}\right)}^{2}\|u\|_{H^{1}\left(\mathbb{R}^{2}\right)}^{\beta} \\
& \leq C(\lambda, \beta)\|u\|_{L^{2}\left(\mathbb{R}^{2}\right)}^{2},
\end{aligned}
$$

and thus

$$
\begin{aligned}
\int_{\mathbb{R}^{2}}\left(e^{\lambda|u|^{2}}-\lambda|u|^{2}-1\right) d x & \leq \lambda \int_{\mathbb{R}^{2}}\left(e^{\lambda|u|^{2}}-1\right)|u|^{2} d x \\
& \leq C(\lambda)\|u\|_{L^{2}\left(\mathbb{R}^{2}\right)}^{2}
\end{aligned}
$$

Lemma 7 (Strichartz estimates). For $s=0$ or 1 ,

$$
2 \leq r, p<\infty, \quad \frac{1}{\gamma(p)}+\frac{1}{p}=\frac{1}{2}, \quad \frac{1}{p}+\frac{1}{p^{\prime}}=1,
$$

(the pairs $(\gamma(p), p)$ were called admissible pairs) we have

$$
\begin{gathered}
\left\|e^{i\left(t-t_{0}\right) \Delta} u\left(t_{0}\right)\right\|_{L^{\gamma(p)}\left(\mathbb{R} ; H_{p}^{s}\right)} \leq C\left\|u\left(t_{0}\right)\right\|_{H^{s}\left(\mathbb{R}^{2}\right)^{\prime}} \\
\left\|\int_{t_{0}}^{t} e^{i(t-\tau) \Delta} f(u(\tau)) d \tau\right\|_{L^{\gamma(p)}\left(\mathbb{R} ; H_{p}^{s}\right)} \leq C\|f(u)\|_{L^{\gamma(r)^{\prime}\left(\mathbb{R} ; H_{r^{\prime}}^{s}\right.}} .
\end{gathered}
$$

Lemma 8 (see [3, Proposition 2.3]). Let $1<r<p<\infty$ be fixed indices. Then for any $q \in[p, \infty)$,

$$
\|u\|_{L^{q}\left(\mathbb{R}^{2}\right)} \leq C(p, r) q^{1 / r^{\prime}+p / r q}\|u\|_{L^{p}\left(\mathbb{R}^{2}\right)}^{p / q}\|u\|_{\dot{H}_{r}^{2 / r}\left(\mathbb{R}^{2}\right)}^{1-p / q} .
$$


As shown in $[6,11]$, to obtain the scattering result, it suffices to show that any finite energy solution has a finite global space-time norm. In fact, if Theorem 3 is true, we have the following theorem.

Lemma 9 (Theorem 3 implies Theorem 4). Let $u$ be a global solution of (5), $H(u) \leq 1$, and $\|u\|_{L_{t, x}^{4}\left(\mathbb{R} \times \mathbb{R}^{2}\right)}<\infty$. Then, for all admissible pairs, we have

$$
\|u\|_{L^{\gamma(p)}\left(\mathbb{R} ; H_{p}^{1}\right)}<\infty .
$$

Moreover, there exist $u_{ \pm} \in H^{1}$ such that

$$
\lim _{t \rightarrow \pm \infty}\left\|u-e^{i t \Delta} u_{ \pm}\right\|_{H^{1}\left(\mathbb{R}^{2}\right)}=0 .
$$

Proof. Defining $X(I)=L^{2 /(1-2 \varepsilon)}\left(I ; H_{1 / \varepsilon}^{1}\right), Y(I)=L^{4}\left(I ; H_{4}^{1}\right)$, by Strichartz estimates, (14) and (15),

$\|u\|_{X(I) \cap Y(I)}$

$$
\begin{aligned}
\leq & C\|u(S)\|_{H^{1}\left(\mathbb{R}^{2}\right)}+C\left\|\lambda|u|^{2} u\right\|_{L^{4 / 3}\left(I ; H_{4 / 3}^{1}\right)} \\
& +C\left\|\left(e^{\lambda|u|^{2}}-\lambda|u|^{2}-1\right) u\right\|_{L^{2 /(1+2 \varepsilon)\left(I ; H_{1 /(1-\varepsilon)}^{1}\right)}} \\
\leq & C(E)+C\|u\|_{L_{t, x}^{4}\left(I \times \mathbb{R}^{2}\right)}^{2}\|u\|_{Y(I)} \\
& +C\|u\|_{L_{t, x}^{4}\left(I \times \mathbb{R}^{2}\right)}^{8 \varepsilon}\|u\|_{X(I)} \\
& \times\left\|\left(e^{\lambda|u|^{2}}-\lambda|u|^{2}-1\right) u^{-8 \varepsilon}\right\|_{L_{t}^{\infty} L_{x}^{1 /(1-4 \varepsilon)}\left(I \times \mathbb{R}^{2}\right)} \\
& +C\|u\|_{L_{t, x}^{4}\left(I \times \mathbb{R}^{2}\right)}^{8 \varepsilon}\|u\|_{X(I)} \\
& \times\left\|\left(e^{\lambda|u|^{2}}-1\right)|u|^{2-8 \varepsilon}\right\|_{L_{t}^{\infty} L_{x}^{1 /(1-4 \varepsilon)}\left(I \times \mathbb{R}^{2}\right)} \\
\leq & C(E)+C(E)\left(\|u\|_{L_{t, x}^{4}\left(I \times \mathbb{R}^{2}\right)}^{2}+\|u\|_{L_{t, x}^{4}\left(I \times \mathbb{R}^{2}\right)}^{8 \varepsilon}\right)\|u\|_{X(I) \cap Y(I)} .
\end{aligned}
$$

Using the same way as in Bourgain [12], one can split $\mathbb{R}$ into finitely many pairwise disjoint intervals:

$$
\mathbb{R}=\bigcup_{j=1}^{J} I_{j}, \quad\|u\|_{L_{t, x}^{4}\left(I_{j} \times \mathbb{R}^{2}\right)} \leq \eta, \quad C(E)\left(\eta^{2}+\eta^{8 \varepsilon}\right) \leq \frac{1}{2} .
$$

By (21),

$$
\|u\|_{L^{2 /(1-2 \varepsilon)}\left(I_{j} ; H_{1 / \varepsilon}^{1}\right) \cap L^{4}\left(I_{j} ; H_{4}^{1}\right)} \leq C(E) .
$$

Since $\|u\|_{L^{\infty}\left(I_{j} ; H^{1}\right)} \leq C(E)$ and $\varepsilon \in(0,1 / 8)$ can be chosen small arbitrarily, by interpolation,

$$
\|u\|_{L^{\gamma(p)}\left(I_{j} ; H_{p}^{1}\right)} \leq C(E),
$$

for all admissible pairs and $j=1,2, \ldots, J$. The desired result (19) follows.
Let

$$
u_{ \pm}=u_{0}-i \int_{0}^{ \pm \infty} e^{-i \tau \Delta} f(u(\tau)) d \tau .
$$

By (19) and (21),

$$
\left\|u_{ \pm}\right\|_{H^{1}\left(\mathbb{R}^{2}\right)} \leqslant 1+\|u\|_{Y(0, \pm \infty)}^{3}+\|u\|_{X(0, \pm \infty) \cap Y(0, \pm \infty)}^{1+8 \varepsilon} \leqslant 1 .
$$

Thus, $u_{ \pm}$were well defined and belong to $H^{1}$. Since

$$
\begin{aligned}
\left\|u-e^{i t \Delta} u_{ \pm}\right\|_{H^{1}\left(\mathbb{R}^{2}\right)} & =\left\|\int_{t}^{ \pm \infty} e^{i(t-\tau) \Delta} f(u(\tau)) d \tau\right\|_{H^{1}\left(\mathbb{R}^{2}\right)} \\
& \leq\|u\|_{Y(t, \pm \infty)}^{3}+\|u\|_{X(t, \pm \infty) \cap Y(t, \pm \infty)}^{1+8 \varepsilon},
\end{aligned}
$$

$\|u\|_{X(0, \pm \infty) \cap Y(0, \pm \infty)} \leqslant 1$

we must have

$$
\begin{aligned}
& \lim _{t \rightarrow \pm \infty}\left\|u-e^{i t \Delta} u_{ \pm}\right\|_{H^{1}\left(\mathbb{R}^{2}\right)} \\
& \quad \lesssim \lim _{t \rightarrow \pm \infty}\left(\|u\|_{Y(t, \pm \infty)}^{3}+\|u\|_{X(t, \pm \infty) \cap Y(t, \pm \infty)}^{1+8 \varepsilon}\right)=0 .
\end{aligned}
$$

(20) was proved.

\section{Stability}

Lemma 10 (stability). For any $A>0$ and $\sigma>0$, there exists $\delta>0$ with the following property: suppose that $u$ : $[0, \infty) \times \mathbb{R}^{2} \rightarrow \mathbb{C}$ satisfies $\|\nabla u\|_{L_{x}^{2}\left(\mathbb{R}^{2}\right)} \leq 1$ for all $t \in[0, \infty)$, $\|u\|_{L_{t, x}^{4}\left([0, \infty) \times \mathbb{R}^{2}\right)} \leq A$ and approximately solves (5) in the sense that

$$
\begin{aligned}
& \left\|\int_{0}^{t} e^{i(t-\tau) \Delta}\left(i u_{t}+\Delta u-f(u)\right)(\tau) d \tau\right\|_{L_{t, x}^{4} \cap L_{t}^{2 /(1-2 \varepsilon)} L_{x}^{1 / \varepsilon}\left([0, \infty) \times \mathbb{R}^{2}\right)} \\
& \quad \leq \delta .
\end{aligned}
$$

Then for any initial data $v(0) \in H^{1}\left(\mathbb{R}^{2}\right)$ satisfying $H(v(0)) \leq 1$ and $\|u(0)-v(0)\|_{L_{x}^{2}\left(\mathbb{R}^{2}\right)} \leq \delta$, there is a unique global solution $v$ to (5) satisfying $\|u-v\|_{L_{t, x}^{4}\left([0, \infty) \times \mathbb{R}^{2}\right)} \leq \sigma$.

Proof. Denote $v=u+w$, then

$$
i w_{t}+\Delta w=(f(u+w)-f(u))-\left(i u_{t}+\Delta u-f(u)\right)
$$

and $\|w(0)\|_{L^{2}\left(\mathbb{R}^{2}\right)} \leq \delta$. Let $X=L_{t}^{\infty} L_{x}^{2} \cap L_{t, x}^{4} \cap L_{t}^{2 /(1-2 \varepsilon)} L_{x}^{1 / \varepsilon}$. By the similar estimates as (21), we have

$$
\begin{aligned}
\|w\|_{X} \leq & \delta+ \\
\leq & 2 \delta+C\left\||u+w|_{0}^{t} e^{i(t-\tau) \Delta}(f(u+w)-f(u))(\tau) d \tau\right\|_{X}+\delta \\
+ & C \|\left(e^{\lambda|u+w|^{2}}-\lambda \|_{L_{t, x}^{4 / 3}}\right. \\
& -\left(e^{\lambda|u|^{2}}-\lambda|u|^{2}-1\right) u \|_{L_{t}^{2 /(1+2 \varepsilon)} L_{x}^{1 /(1-\varepsilon)}}
\end{aligned}
$$




$$
\begin{aligned}
& \leq 2 \delta+C\left\||u+w|^{2}|w|+|u|^{2}|w|\right\|_{L_{t, x}^{4 / 3}} \\
& +C \|\left(e^{\lambda|u+w|^{2}}-\lambda|u+w|^{2}-1\right)|w| \\
& +\left(e^{\lambda|u|^{2}}-\lambda|u|^{2}-1\right)|w| \|_{L_{t}^{2 /(1+2 \varepsilon)} L_{x}^{1 /(1-\varepsilon)}} \\
& +C \|\left(e^{\lambda|u+w|^{2}}-1\right)|u+w|^{2}|w| \\
& +\left(e^{\lambda|u|^{2}}-1\right)|u|^{2}|w| \|_{L_{t}^{2 /(1+2 \varepsilon)} L_{x}^{1 /(1-\varepsilon)}} \\
& \leq 2 \delta+C\left(\|u\|_{L_{t, x}^{4}}^{2}+\|u+w\|_{L_{t, x}^{4}}^{2}\right)\|w\|_{L_{t, x}^{4}} \\
& +C\left(\|u\|_{L_{t, x}^{4}}^{8 \varepsilon}+\|u+w\|_{L_{t, x}^{4}}^{8 \varepsilon}\right)\|w\|_{L_{t}^{2 /(1-2 \varepsilon)} L_{x}^{1 / \varepsilon}} \\
& \leq 2 \delta+C\left(\|u\|_{L_{t, x}^{4}}^{2}+\|u\|_{L_{t, x}^{4}}^{8 \varepsilon}\right)\|w\|_{X}+\|w\|_{X}^{1+8 \varepsilon}+\|w\|_{X}^{3} .
\end{aligned}
$$

Then we subdivide the time interval $[0, \infty)$ into finite subintervals $I_{j}=\left[t_{j}, t_{j+1}\right], j=1, \ldots, J, J=J(A)$ such that

$$
C\left(\|u\|_{L_{t, x}^{4}\left(I_{j} \times \mathbb{R}^{2}\right)}^{2}+\|u\|_{L_{t, x}^{4}\left(I_{j} \times \mathbb{R}^{2}\right)}^{8 \varepsilon}\right)<\frac{1}{4}
$$

for each $j$. Let $\delta=\delta(A, J)$ be small such that

$$
C 8^{2 J} \delta \ll 1, \quad 8^{2 J} \delta \ll \sigma .
$$

Then by (31) on $I_{1} \times \mathbb{R}^{2}$, we have $\|w\|_{X\left(I_{1} \times \mathbb{R}^{2}\right)} \leq 8 \delta$ and

$$
\begin{aligned}
& \left\|w\left(t_{2}\right)\right\|_{L_{x}^{2}\left(\mathbb{R}^{2}\right)} \\
& \leq\left\|e^{i\left(t_{2}-t_{1}\right) \Delta} w\left(t_{1}\right)\right\|_{L_{x}^{2}\left(\mathbb{R}^{2}\right)} \\
& \quad+\left\|\int_{t_{1}}^{t_{2}} e^{i\left(t_{2}-\tau\right) \Delta}(f(u+w)-f(u))(\tau) d \tau\right\|_{L_{x}^{2}\left(\mathbb{R}^{2}\right)}+\delta \\
& \leq 2 \delta+\delta+C\left(\|u\|_{L_{t, x}^{4}\left(I_{1} \times \mathbb{R}^{2}\right)}^{2}+\|u\|_{L_{t, x}^{4}\left(I_{1} \times \mathbb{R}^{2}\right)}^{8 \varepsilon}\right)\|w\|_{X\left(I_{1} \times \mathbb{R}^{2}\right)} \\
& \quad+\|w\|_{X\left(I_{1} \times \mathbb{R}^{2}\right)}^{1+8 \varepsilon}+\|w\|_{X\left(I_{1} \times \mathbb{R}^{2}\right)}^{3} \\
& \leq 2 \delta+4 \cdot 8 \delta \leq 8^{2} \delta .
\end{aligned}
$$

Using the same analysis as above, we can get $\|w\|_{X\left(I_{2} \times \mathbb{R}^{2}\right)} \leq$ $8^{3} \delta$. Iterating this for $I_{2}, I_{3}, \ldots, I_{J}$, we obtain $\|w\|_{X\left([0, \infty) \times \mathbb{R}^{2}\right)} \leq$ $8^{2 J} \delta \leq \sigma$; the desired result was obtained.

\section{Linear Profile Decomposition}

In this section, we will give the linear profile decomposition for Schrödinger equation in $H^{1}\left(\mathbb{R}^{2}\right)$. First, we give some definitions and lemmas.

Definition 11 (symmetry group, [13]). For any phase $\theta \epsilon$ $\mathbb{R} / 2 \pi \mathbb{Z}$, position $x_{0} \in \mathbb{R}^{2}$, frequency $\xi_{0} \in \mathbb{R}^{2}$, and scaling parameter $\lambda>0$, we define the unitary transformation $g_{\theta, \xi_{0}, x_{0}, \lambda}: L_{x}^{2}\left(\mathbb{R}^{2}\right) \rightarrow L_{x}^{2}\left(\mathbb{R}^{2}\right)$ by the formula

$$
g_{\theta, \xi_{0}, x_{0}, \lambda} f(x):=\frac{1}{\lambda} e^{i \theta} e^{i x \cdot \xi_{0}} f\left(\frac{x-x_{0}}{\lambda}\right) .
$$

We let $G$ be the collection of such transformations; this is a group with identity $g_{0,0,0,1}$, inverse $g_{\theta, \xi_{0}, x_{0}, \lambda}^{-1}=$ $g_{-\theta-x_{0}} \xi_{0},-\lambda \xi_{0},-x_{0} / \lambda, 1 / \lambda$, and group law

$$
g_{\theta, \xi_{0}, x_{0}, \lambda} g_{\theta^{\prime}, \xi_{0}^{\prime}, x_{0}^{\prime}, \lambda^{\prime}}=g_{\theta+\theta^{\prime}-x_{0} \xi_{0}^{\prime} / \lambda, \xi_{0}+\xi_{0}^{\prime} / \lambda, x_{0}+\lambda x_{0}^{\prime}, \lambda \lambda^{\prime}}
$$

If $u: I \times \mathbb{R}^{2} \rightarrow \mathbb{C}$ is a function, we define $T_{g_{\theta, \xi_{0}, x_{0}, \lambda}} u:$ $\lambda^{2} I \times \mathbb{R}^{2} \rightarrow \mathbb{C}$, where $\lambda^{2} I:=\left\{\lambda^{2} t: t \in I\right\}$ by the formula

$$
\left(T_{g_{\theta, \xi_{0}, x_{0}, \lambda}} u\right)(t, x):=\frac{1}{\lambda} e^{i \theta} e^{i x \cdot \xi_{0}} e^{-i t\left|\xi_{0}\right|^{2}} u\left(\frac{t}{\lambda^{2}}, \frac{x-x_{0}-2 \xi_{0} t}{\lambda}\right),
$$

or equivalently

$$
\left(T_{g_{\theta, \xi_{0}, x_{0}, \lambda}} u\right)(t)=g_{\theta-t\left|\xi_{0}\right|^{2}, \xi_{0}, x_{0}+2 \xi_{0} t, \lambda}\left(u\left(\frac{t}{\lambda^{2}}\right)\right) .
$$

If $g \in G$, we can easily prove that $M\left(T_{g} u\right)=M(u)$ and $S\left(T_{g} u\right)=S(u)$.

Definition 12 (enlarged group, [13]). For any phase $\theta \epsilon$ $\mathbb{R} / 2 \pi \mathbb{Z}$, position $x_{0} \in \mathbb{R}^{2}$, frequency $\xi_{0} \in \mathbb{R}^{2}$, scaling parameter $\lambda>0$, and time $t_{0}$, we define the unitary transformation $g_{\theta, \xi_{0}, x_{0}, \lambda, t_{0}}: L_{x}^{2}\left(\mathbb{R}^{2}\right) \rightarrow L_{x}^{2}\left(\mathbb{R}^{2}\right)$ by the formula

$$
g_{\theta, \xi_{0}, x_{0}, \lambda, t_{0}}=g_{\theta, \xi_{0}, x_{0}, \lambda} e^{i t_{0} \Delta}
$$

or in other words

$$
g_{\theta, \xi_{0}, x_{0}, \lambda, t_{0}} f(x):=\frac{1}{\lambda} e^{i \theta} e^{i x \cdot \xi_{0}}\left(e^{i t_{0} \Delta} f\right)\left(\frac{x-x_{0}}{\lambda}\right) .
$$

Let $G^{\prime}$ be the collection of such transformations. We also let $G^{\prime}$ act on global space-time function $u: \mathbb{R} \times \mathbb{R}^{2} \rightarrow \mathbb{C}$ by defining

$$
\begin{aligned}
& T_{g_{\theta, \xi_{0}, x_{0}, \lambda, t_{0}}} u(t, x) \\
& \quad:=\frac{1}{\lambda} e^{i \theta} e^{i x \cdot \xi_{0}} e^{-i t\left|\xi_{0}\right|^{2}}\left(e^{i t_{0} \Delta} u\right)\left(\frac{t}{\lambda^{2}}, \frac{x-x_{0}-2 \xi_{0} t}{\lambda}\right),
\end{aligned}
$$

or equivalently

$$
\left(T_{g_{\theta, \xi_{0}, x_{0}, \lambda, t_{0}}} u\right)(t)=g_{\theta-t\left|\xi_{0}\right|^{2}, \xi_{0}, x_{0}+2 \xi_{0} t, \lambda, t_{0}}\left(u\left(\frac{t}{\lambda^{2}}\right)\right) .
$$

Lemma 13 (linear profiles for $L^{2}$ sequence, [14]). Let $u_{n}$ be a bounded sequence in $L_{x}^{2}\left(\mathbb{R}^{2}\right)$. Then (after passing to a subsequence if necessary) there exists a family $\phi^{(j)}, j=1,2, \ldots$ of functions in $L_{x}^{2}\left(\mathbb{R}^{2}\right)$ and group elements $g_{n}^{(j)} \in G^{\prime}$ for $j, n=$ $1,2, \ldots$ such that one has the decomposition

$$
u_{n}=\sum_{j=1}^{l} g_{n}^{(j)} \phi^{(j)}+w_{n}^{(l)}
$$


for all $l=1,2, \ldots$; here, $w_{n}^{(l)} \in L_{x}^{2}\left(\mathbb{R}^{2}\right)$ is such that its linear evolution has asymptotically vanishing scattering size:

$$
\lim _{l \rightarrow \infty} \limsup _{n \rightarrow \infty} S\left(e^{i t \Delta} w_{n}^{(l)}\right)=0
$$

Moreover, for any $j \neq j^{\prime}$,

$$
\begin{aligned}
& \frac{\lambda_{n}^{\left(j^{\prime}\right)}}{\lambda_{n}^{(j)}}+ \frac{\lambda_{n}^{(j)}}{\lambda_{n}^{\left(j^{\prime}\right)}}+\lambda_{n}^{(j)} \lambda_{n}^{\left(j^{\prime}\right)}\left|\xi_{n}^{(j)}-\xi_{n}^{\left(j^{\prime}\right)}\right|^{2}+\frac{\left|x_{n}^{(j)}-x_{n}^{\left(j^{\prime}\right)}\right|^{2}}{\lambda_{n}^{(j)} \lambda_{n}^{\left(j^{\prime}\right)}} \\
&+\frac{\left|t_{n}^{(j)}\left(\lambda_{n}^{(j)}\right)^{2}-t_{n}^{\left(j^{\prime}\right)}\left(\lambda_{n}^{\left(j^{\prime}\right)}\right)^{2}\right|}{\lambda_{n}^{(j)} \lambda_{n}^{\left(j^{\prime}\right)}} \longrightarrow \infty .
\end{aligned}
$$

Furthermore, for any $l \geq 1$, one has the mass decoupling property

$$
\lim _{n \rightarrow \infty}\left[M\left(u_{n}\right)-\sum_{j=1}^{l} M\left(\phi^{(j)}\right)-M\left(w_{n}^{(l)}\right)\right]=0 .
$$

For any $j \leq l$, we have

$$
\left(g_{n}^{(j)}\right)^{-1} w_{n}^{(l)} \longrightarrow 0 \text { weakly in } L_{x}^{2}\left(\mathbb{R}^{2}\right) .
$$

Remark 14. If the orthogonal condition (45) holds, then (see [14])

$$
\begin{gathered}
\lim _{n \rightarrow \infty}\left\langle g_{n}^{(j)} \phi^{(j)}, g_{n}^{\left(j^{\prime}\right)} \phi^{\left(j^{\prime}\right)}\right\rangle_{L^{2}\left(\mathbb{R}^{2}\right)}=0, \quad j \neq j^{\prime}, \\
\lim _{n \rightarrow \infty}\left\langle g_{n}^{(j)} \phi^{(j)}, w_{n}^{(l)}\right\rangle_{L^{2}\left(\mathbb{R}^{2}\right)}=0 .
\end{gathered}
$$

Moreover, if $v^{(j)}, v^{\left(j^{\prime}\right)} \in L_{t, x}^{4}\left(\mathbb{R} \times \mathbb{R}^{2}\right)$, then (see $\left.[14,15]\right)$, for any $0<\theta<1$,

$$
\lim _{n \rightarrow \infty}\left\|\left|T_{g_{n}^{(j)}} v^{(j)}\right|^{1-\theta}\left|T_{g_{n}^{\left(j^{\prime}\right)}} v^{\left(j^{\prime}\right)}\right|^{\theta}\right\|_{L_{t, x}^{4}\left(\mathbb{R} \times \mathbb{R}^{2}\right)}=0 .
$$

If $v^{(1)}, \ldots, v^{(l)} \in L_{t, x}^{4}\left(\mathbb{R} \times \mathbb{R}^{2}\right)$, then (see [16, Lemma 5.5])

$$
\lim _{n \rightarrow \infty} S\left(\sum_{j=1}^{l} g_{n}^{(j)} v^{(j)}\right) \leq \sum_{j=1}^{l} S\left(v^{(j)}\right)
$$

Remark 15. As each linear profile $\phi^{(j)}$ in Lemma 13 is constructed in the sense that

$$
e^{i t_{n}^{(j)} \Delta}\left\{e^{i \xi_{n}^{(j)} \cdot x} \lambda_{n}^{(j)} u_{n}\left(\lambda_{n}^{(j)} x\right)\right\}\left(x+x_{n}^{(j)}\right) \longrightarrow \phi^{(j)}
$$

weakly in $L_{x}^{2}\left(\mathbb{R}^{2}\right)$ (see [14]), after passing to a subsequence in $n$, rearrangement, translation, and refining $\phi^{(j)}$ accordingly, we may assume that the parameters satisfy the following properties:

(i) $t_{n}^{(j)} \rightarrow \pm \infty$ as $n \rightarrow \infty$, or $t_{n}^{(j)} \equiv 0$ for all $n, j$;

(ii) $\lambda_{n}^{(j)} \rightarrow 0$ or $\infty$ as $n \rightarrow \infty$, or $\lambda_{n}^{(j)} \equiv 1$ for all $n, j$; (iii) $\left|\xi_{n}^{(j)}\right| \rightarrow \infty$ as $n \rightarrow \infty$, or $\xi_{n}^{(j)} \equiv \xi^{(j)}$ with $\left|\xi^{(j)}\right|<\infty$;

(iv) when $\lambda_{n}^{(j)} \equiv 1, \xi_{n}^{(j)} \equiv \xi^{(j)}$ and $\left|\xi^{(j)}\right|<\infty$, we can let $\xi^{(j)} \equiv 0$.

Our main result in this section is the following lemma.

Lemma 16 (linear profiles for $H^{1}$ sequence). Let $u_{n}$ be a bounded sequence in $H^{1}\left(\mathbb{R}^{2}\right)$. Then up to a subsequence, for any $J \geq 1$, there exists a sequence $\phi_{\alpha}^{J}$ in $H^{1}\left(\mathbb{R}^{2}\right)$ and a sequence

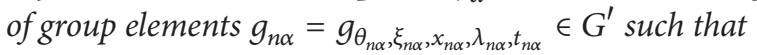

$$
u_{n}=\sum_{\alpha=1}^{J} g_{n \alpha} \phi_{\alpha}^{J}+R(n, J)
$$

Here, for each $\alpha, \lambda_{n \alpha}$ and $\xi_{n \alpha}$ must satisfy

$$
\text { " } \lambda_{n \alpha} \equiv 1 \text { and } \xi_{n \alpha} \equiv 0 \text {," or " } \lambda_{n \alpha} \longrightarrow \infty \text {." }
$$

$R(n, J) \in H^{1}\left(\mathbb{R}^{2}\right)$ is such that

$$
\lim _{J \rightarrow \infty} \limsup _{n \rightarrow \infty} S\left(e^{i t \Delta} R(n, J)\right)=0 .
$$

Moreover, for any $\alpha \neq \alpha^{\prime}$, one has the same orthogonal conditions as (45). For any $J \geq 1$, one has the following decoupling properties:

$$
\lim _{n \rightarrow \infty}\left\{\left\|u_{n}\right\|_{L^{2}\left(\mathbb{R}^{2}\right)}^{2}-\sum_{\alpha=1}^{J}\left\|\phi_{\alpha}^{J}\right\|_{L^{2}\left(\mathbb{R}^{2}\right)}^{2}-\|R(n, J)\|_{L^{2}\left(\mathbb{R}^{2}\right)}^{2}\right\}=0,
$$

$$
\begin{aligned}
& \lim _{n \rightarrow \infty}\left\{\left\|u_{n}\right\|_{\dot{H}^{1}\left(\mathbb{R}^{2}\right)}^{2}-\sum_{\alpha=1}^{J}\left\|g_{n \alpha} \phi_{\alpha}^{J}\right\|_{\dot{H}^{1}\left(\mathbb{R}^{2}\right)}^{2}-\|R(n, J)\|_{\dot{H}^{1}\left(\mathbb{R}^{2}\right)}^{2}\right\} \\
& \quad=0
\end{aligned}
$$

$$
\lim _{J \rightarrow \infty} \limsup _{n \rightarrow \infty}\left\{H\left(u_{n}\right)-\sum_{\alpha=1}^{J} H\left(g_{n \alpha} \phi_{\alpha}^{J}\right)-H(R(n, J))\right\}=0 .
$$

Proof. Let

$$
\square_{k}=\mathscr{F}^{-1} \chi_{k} \mathscr{F}, \quad \chi_{k}= \begin{cases}1 & 2^{k-1}<|\xi| \leq 2^{k}, \\ 0 & \text { else. }\end{cases}
$$

Then, we have

$$
\begin{gathered}
u_{n}=\sum_{k=-\infty}^{+\infty} \square_{k} u_{n}:=\sum_{|k| \leq N} \square_{k} u_{n}+R_{N}, \\
\left\|u_{n}\right\|_{L^{2}\left(\mathbb{R}^{2}\right)}^{2}=\sum_{|k| \leq N}\left\|\square_{k} u_{n}\right\|_{L^{2}\left(\mathbb{R}^{2}\right)}^{2}+\left\|R_{N}\right\|_{L^{2}\left(\mathbb{R}^{2}\right)}^{2}, \\
\left\|u_{n}\right\|_{\dot{H}^{1}\left(\mathbb{R}^{2}\right)}^{2}=\sum_{|k| \leq N}\left\|\square_{k} u_{n}\right\|_{\dot{H}^{1}\left(\mathbb{R}^{2}\right)}^{2}+\left\|R_{N}\right\|_{\dot{H}^{1}\left(\mathbb{R}^{2}\right)}^{2}, \\
\lim _{N \rightarrow \infty} \limsup _{n \rightarrow \infty}\left\|R_{N}\right\|_{L^{2}\left(\mathbb{R}^{2}\right)}=0 .
\end{gathered}
$$


By Lemma 13, after passing to a subsequence if necessary, we can obtain

$$
\square_{k} u_{n}=\sum_{j=1}^{l_{k}} g_{n k}^{(j)} \phi_{k}^{(j)}+w_{n k}^{\left(l_{k}\right)}
$$

with the stated properties (i)-(iv) in Remark 15 and (43)(47). Denote

$$
\Lambda_{1,0}=\left\{(k, j)|| k \mid \leq N, 1 \leq j \leq l_{k}, \lambda_{n k}^{(j)} \equiv 1, \xi_{n k}^{(j)} \equiv 0\right\},
$$$$
\Lambda_{1, \infty}=\left\{(k, j)|| k\left|\leq N, 1 \leq j \leq l_{k}, \lambda_{n k}^{(j)} \equiv 1,\right| \xi_{n k}^{(j)} \mid \longrightarrow \infty\right\},
$$

$$
\Lambda_{0}=\left\{(k, j)|| k \mid \leq N, 1 \leq j \leq l_{k}, \lambda_{n k}^{(j)} \longrightarrow 0\right\},
$$$$
\Lambda_{\infty, 0}=\left\{(k, j)|| k \mid \leq N, 1 \leq j \leq l_{k}, \lambda_{n k}^{(j)} \longrightarrow \infty, \xi_{n k}^{(j)} \equiv \xi_{k}^{(j)},\right.
$$

$$
\left.\left|\xi_{k}^{(j)}\right|<2^{k-1}\right\}
$$

$\Lambda_{\infty, 1}=\left\{(k, j)|| k \mid \leq N, 1 \leq j \leq l_{k}, \lambda_{n k}^{(j)} \longrightarrow \infty, \xi_{n k}^{(j)} \equiv \xi_{k}^{(j)}\right.$,

$$
\begin{aligned}
& \left.\left|\xi_{k}^{(j)}\right| \in\left[2^{k-1}, 2^{k}\right]\right\}, \\
\Lambda_{\infty, \infty}= & \left\{(k, j)|| k\left|\leq N, 1 \leq j \leq l_{k}, \lambda_{n k}^{(j)} \longrightarrow \infty,\right| \xi_{n k}^{(j)} \mid \longrightarrow \infty\right. \\
& \text { or } \left.\xi_{n k}^{(j)} \equiv \xi_{k}^{(j)},\left|\xi_{k}^{(j)}\right|>2^{k}\right\} .
\end{aligned}
$$

Step 1. We prove that

$$
u_{n}=\sum_{(k, j) \in \Lambda_{1,0} \cup \Lambda_{\infty, 1}} g_{n k}^{(j)} \phi_{k}^{(j)}+R
$$

with $\phi_{k}^{(j)} \in H^{1}$ and for each fixed $N$,

$$
\begin{gathered}
\lim _{n \rightarrow \infty}\left\{\left\|u_{n}\right\|_{L^{2}\left(\mathbb{R}^{2}\right)}^{2}-\sum_{(k, j) \in \Lambda_{1,0} \cup \Lambda_{\infty, 1}}\left\|\phi_{k}^{(j)}\right\|_{L^{2}\left(\mathbb{R}^{2}\right)}^{2}\right. \\
\left.-\|R\|_{L^{2}\left(\mathbb{R}^{2}\right)}^{2}\right\}=0, \\
\lim _{n \rightarrow \infty}\left\{\left\|u_{n}\right\|_{\dot{H}^{1}\left(\mathbb{R}^{2}\right)}^{2} \sum_{(k, j) \in \Lambda_{1,0} \cup \Lambda_{\infty, 1}}\left\|g_{n k}^{(j)} \phi_{k}^{(j)}\right\|_{\dot{H}^{1}\left(\mathbb{R}^{2}\right)}^{2}\right. \\
\left.-\|R\|_{\dot{H}^{1}\left(\mathbb{R}^{2}\right)}^{2}\right\}=0, \\
\lim _{N \rightarrow \infty} \lim _{l_{k} \rightarrow \infty} \limsup _{n \rightarrow \infty} S\left(e^{i t \triangle} R\right)=0,
\end{gathered}
$$

where

$$
R=R_{N}+R_{w}, \quad R_{w}=\sum_{|k| \leq N} w_{n k}^{\left(l_{k}\right)}
$$

By (44) and $\lim _{N \rightarrow \infty} \lim \sup _{n \rightarrow \infty}\left\|R_{N}\right\|_{L^{2}\left(\mathbb{R}^{2}\right)}=0$, (64) holds obviously. For (62), we prove it by induction. For every $k$, suppose that

$$
\square_{k} u_{n}=g_{n k}^{(1)} \phi_{k}^{(1)}+w_{n k}^{(1)}
$$

Case 1. If $(k, 1) \in \Lambda_{1, \infty} \cup \Lambda_{0} \cup \Lambda_{\infty, 0} \cup \Lambda_{\infty, \infty}$, we have $\phi_{k}^{(1)}=0$. In fact, by (66),

$$
\phi_{k}^{(1)}=\left(g_{n k}^{(1)}\right)^{-1} \square_{k} u_{n}-\left(g_{n k}^{(1)}\right)^{-1} w_{n k}^{(1)} .
$$

Thus,

$$
\begin{aligned}
\left\|\phi_{k}^{(1)}\right\|_{L^{2}\left(\mathbb{R}^{2}\right)}^{2}= & \left\langle\left(g_{n k}^{(1)}\right)^{-1} \square_{k} u_{n}-\left(g_{n k}^{(1)}\right)^{-1} w_{n k}^{(1)}, \phi_{k}^{(1)}\right\rangle_{L^{2}\left(\mathbb{R}^{2}\right)} \\
= & \left\langle u_{n}, \square_{k} g_{n k}^{(1)} \phi_{k}^{(1)}\right\rangle_{L^{2}\left(\mathbb{R}^{2}\right)} \\
& -\left\langle\left(g_{n k}^{(1)}\right)^{-1} w_{n k}^{(1)}, \phi_{k}^{(1)}\right\rangle_{L^{2}\left(\mathbb{R}^{2}\right)} .
\end{aligned}
$$

Using (47),

$$
\left\langle\left(g_{n k}^{(1)}\right)^{-1} w_{n k}^{(1)}, \phi_{k}^{(1)}\right\rangle_{L^{2}\left(\mathbb{R}^{2}\right)} \longrightarrow 0 \quad \text { as } n \longrightarrow \infty .
$$

By direct calculation,

$$
\begin{aligned}
\square_{k} g_{n k}^{(1)} & \phi_{k}^{(1)} \\
= & \mathscr{F}^{-1} \chi_{k}(\xi) \lambda_{n k}^{(1)} e^{i \theta_{n k}^{(1)}} e^{-i x_{n k}^{(1)}\left(\xi+\xi_{n k}^{(1)}\right)} e^{-i t_{n k}^{(1)}\left(\lambda_{n k}^{(1)}\right)^{2}\left|\xi+\xi_{n k}^{(1)}\right|^{2}} \\
& \times \widehat{\phi_{k}^{(1)}}\left(\lambda_{n k}^{(1)}\left(\xi+\xi_{n k}^{(1)}\right)\right) \\
= & \frac{1}{\lambda_{n k}^{(1)}} e^{i \theta_{n k}^{(1)}} e^{i x \cdot \xi_{n k}^{(1)}}\left(\mathscr{F}^{-1} \chi_{k}\left(\frac{\xi}{\lambda_{n k}^{(1)}}+\xi_{n k}^{(1)}\right) e^{-i t_{n k}^{(1)}|\xi|^{2}} \widehat{\phi_{k}^{(1)}}\right) \\
& \times\left(\frac{x-x_{n k}^{(1)}}{\lambda_{n k}^{(1)}}\right) .
\end{aligned}
$$

Let $n \rightarrow \infty$. When $(k, 1) \in \Lambda_{1, \infty}$,

$$
\left\|\square_{k} g_{n k}^{(1)} \phi_{k}^{(1)}\right\|_{L^{2}\left(\mathbb{R}^{2}\right)}^{2} \leq \int_{2^{k-1} \leq\left|\xi+\xi_{n k}^{(1)}\right| \leq 2^{k}}\left|\mathscr{F} \phi_{k}^{(1)}\right|^{2} d \xi \longrightarrow 0 .
$$

When $(k, 1) \in \Lambda_{0}$,

$$
\left\|\square_{k} g_{n k}^{(1)} \phi_{k}^{(1)}\right\|_{L^{2}\left(\mathbb{R}^{2}\right)}^{2} \leq \int_{\lambda_{n k}^{(1)} k^{k-1} \leq\left|\xi+\lambda_{n k}^{(1)} \xi_{n k}^{(1)}\right| \leq \lambda_{n k}^{(1)} 2^{k}}\left|\mathscr{F} \phi_{k}^{(1)}\right|^{2} d \xi \longrightarrow 0 .
$$

When $(k, 1) \in \Lambda_{\infty, 0}$,

$$
\left\|\square_{k} g_{n k}^{(1)} \phi_{k}^{(1)}\right\|_{L^{2}\left(\mathbb{R}^{2}\right)}^{2} \leq \int_{|\xi| \geq \lambda_{n k}^{(1)}\left(2^{k-1}-\left|\xi_{k}^{(1)}\right|\right)}\left|\mathscr{F} \phi_{k}^{(1)}\right|^{2} d \xi \longrightarrow 0 .
$$


When $(k, 1) \in \Lambda_{\infty, \infty}$,

$$
\left\|\square_{k} g_{n k}^{(1)} \phi_{k}^{(1)}\right\|_{L^{2}\left(\mathbb{R}^{2}\right)}^{2} \leq \int_{|\xi| \geq \lambda_{n k}^{(1)}\left(\left|\xi_{n k}^{(1)}\right|-2^{k}\right)}\left|\mathscr{F} \phi_{k}^{(1)}\right|^{2} d \xi \longrightarrow 0 .
$$

By (68)-(74), $\left\|\phi_{k}^{(1)}\right\|_{L^{2}\left(\mathbb{R}^{2}\right)}^{2}=0$ and thus $\phi_{k}^{(1)}=0$.

Case 2. If $(k, 1) \in \Lambda_{1,0} \cup \Lambda_{\infty, 1}$, we can prove

$$
\left\|g_{n k}^{(1)} \phi_{k}^{(1)}-\square_{k} g_{n k}^{(1)} \phi_{k}^{(1)}\right\|_{L^{2}\left(\mathbb{R}^{2}\right)} \longrightarrow 0 \quad \text { as } n \longrightarrow \infty .
$$

By absorbing the error into $w_{n k}^{(1)}$, we can suppose $g_{n k}^{(1)} \phi_{k}^{(1)}=$ $\square_{k} g_{n k}^{(1)} \phi_{k}^{(1)}$. Since $\square_{k} g_{n k}^{(1)} \phi_{k}^{(1)} \in H^{1}$ for each fixed $n$, we must have $\phi_{k}^{(1)} \in H^{1}$.

Now, we begin to prove (75). Let $\chi_{A_{k}^{(1)}}$ be the characteristic function of the set $A_{k}^{(1)}$ and $P_{A_{k}^{(1)}}=\mathscr{F}^{-1} \chi_{A_{k}^{(1)}} \mathscr{F}$, and then

$$
\begin{aligned}
g_{n k}^{(1)}\left(P_{A_{k}^{(1)}} \phi_{k}^{(1)}+P_{A_{k}^{(1)}}\left(g_{n k}^{(1)}\right)^{-1} w_{n k}^{(1)}\right) & =P_{\widetilde{A}_{k}^{(1)}}\left(g_{n k}^{(1)} \phi_{k}^{(1)}+w_{n k}^{(1)}\right) \\
& =P_{\widetilde{A}_{k}^{(1)}} \square_{k} u_{n}
\end{aligned}
$$

where

$$
P_{\widetilde{A}_{k}^{(1)}}=\mathscr{F}^{-1} \chi_{A_{k}^{(1)}}\left(\lambda_{n k}^{(1)}\left(\xi-\xi_{n k}^{(1)}\right)\right) \mathscr{F} .
$$

Note that

$$
\begin{aligned}
& \left\langle P_{A_{k}^{(1)}} \phi_{k}^{(1)}, P_{A_{k}^{(1)}}\left(g_{n k}^{(1)}\right)^{-1} w_{n k}^{(1)}\right\rangle_{L^{2}\left(\mathbb{R}^{2}\right)} \\
& =\left\langle P_{A_{k}^{(1)}} \phi_{k}^{(1)},\left(g_{n k}^{(1)}\right)^{-1} w_{n k}^{(1)}\right\rangle_{L^{2}\left(\mathbb{R}^{2}\right)} \longrightarrow 0 .
\end{aligned}
$$

We have

$$
\begin{gathered}
\lim _{n \rightarrow \infty}\left(\left\|P_{\widetilde{A}_{k}^{(1)}} \square_{k} u_{n}\right\|_{L^{2}\left(\mathbb{R}^{2}\right)}^{2}-\left\|P_{A_{k}^{(1)}} \phi_{k}^{(1)}\right\|_{L^{2}\left(\mathbb{R}^{2}\right)}^{2}\right. \\
\left.-\left\|P_{A_{k}^{(1)}}\left(g_{n k}^{(1)}\right)^{-1} w_{n k}^{(1)}\right\|_{L^{2}\left(\mathbb{R}^{2}\right)}^{2}\right)=0 .
\end{gathered}
$$

When $(k, 1) \in \Lambda_{1,0}$, we have $P_{A_{k}^{(1)}}=P_{\widetilde{A}_{k}^{(1)}}$. Choosing $A_{k}^{(1)}=$ $\left\{\xi|| \xi \mid \leq 2^{k-1}\right.$ or $\left.|\xi|>2^{k}\right\}$, then by $(79), P_{A_{k}^{(1)}} \phi_{k}^{(1)}=0$, the desired result follows.

When $(k, 1) \in \Lambda_{\infty, 1}$ and $\left|\xi_{k}^{(1)}\right| \in\left(2^{k-1}, 2^{k}\right)$, we have

$$
\begin{aligned}
& \left\|g_{n k}^{(1)} \phi_{k}^{(1)}-\square_{k} g_{n k}^{(1)} \phi_{k}^{(1)}\right\|_{L^{2}\left(\mathbb{R}^{2}\right)}^{2} \\
& \leq \int_{\left|\xi+\lambda_{n k}^{(1)} \xi_{k}^{(1)}\right| \leq \lambda_{n k}^{(1)} 2^{k-1}}\left|\mathscr{F} \phi_{k}^{(1)}\right|^{2} d \xi \\
& \quad+\int_{\left|\xi+\lambda_{n k}^{(1)} \xi_{k}^{(1)}\right| \geq \lambda_{n k}^{(1)} 2^{k}}\left|\mathscr{F} \phi_{k}^{(1)}\right|^{2} d \xi \\
& \leq \int_{|\xi| \geq \lambda_{n k}^{(1)}\left(\left|\xi_{k}^{(1)}\right|-2^{k-1}\right)}\left|\mathscr{F} \phi_{k}^{(1)}\right|^{2} d \xi \\
& \quad+\int_{|\xi| \geq \lambda_{n k}^{(1)}\left(2^{k}-\left|\xi_{k}^{(1)}\right|\right)}\left|\mathscr{F} \phi_{k}^{(1)}\right|^{2} d \xi \longrightarrow 0 \\
& \text { as } n \longrightarrow \infty .
\end{aligned}
$$

When $(k, 1) \in \Lambda_{\infty, 1}$ and $\left|\xi_{k}^{(1)}\right|=2^{k}$, we denote $\xi=\left(\xi_{1}, \xi_{2}\right)$ and $\xi_{k}^{(1)}=\left(\xi_{k 1}^{(1)}, \xi_{k 2}^{(1)}\right)$. The line $\xi_{2}=-\left(\xi_{k 1}^{(1)} / \xi_{k 2}^{(1)}\right) \xi_{1}$ (when $\xi_{k 2}^{(j)}=$ 0 , we use the line $\xi_{1}=0$ instead) separates the frequency space $\mathbb{R}^{2}$ into two half-planes. We let $A_{k}^{(1)}$ to be the half-plane which contains the point $\xi_{k}^{(1)}$, and then

$$
P_{\widetilde{A}_{k}^{(1)}} \square_{k} u_{n}=\mathscr{F}^{-1} \chi_{A_{k}^{(1)}}\left(\lambda_{n k}^{(1)}\left(\xi-\xi_{k}^{(1)}\right)\right) \chi_{k} \mathscr{F} u_{n}=0 .
$$

By (79), we have $P_{A_{k}^{(1)}} \phi_{k}^{(1)}=0$. Note that

$$
\begin{aligned}
& \lim _{n \rightarrow \infty}\left\|g_{n k}^{(1)}\left(I-P_{A_{k}^{(1)}}\right) \phi_{k}^{(1)}-\square_{k} g_{n k}^{(1)} \phi_{k}^{(j)}\right\|_{L^{2}\left(\mathbb{R}^{2}\right)}^{2} \\
& \quad=\lim _{n \rightarrow \infty}\left\|\left[\left(1-\chi_{A_{k}^{(1)}}\right)(\cdot)-\chi_{k}\left(\frac{\cdot}{\lambda_{n k}^{(1)}}+\xi_{k}^{(1)}\right)\right] \mathscr{F} \phi_{k}^{(1)}\right\|_{L^{2}\left(\mathbb{R}^{2}\right)}^{2} \\
& \quad \leq \lim _{n \rightarrow \infty} \int_{\xi \in \mathbb{R}^{2} \backslash\left(A_{k}^{(1)} \cup\left\{2^{k-1} \leq\left|\xi / \lambda_{n k}^{(1)}+\xi_{k}^{(1)}\right| \leq 2^{k}\right\}\right)}\left|\mathscr{F} \phi_{k}^{(1)}\right|^{2} d \xi \\
& \quad=0 .
\end{aligned}
$$

(75) holds.

When $(k, 1) \in \Lambda_{\infty, 1}$ and $\left|\xi_{k}^{(1)}\right|=2^{k-1}$, let $A_{k}^{(1)}$ be the halfplane which does NOT contain the point $\xi_{k}^{(1)}$; we can prove (75) similarly as above.

By the proof above, we get

$$
\square_{k} u_{n}=g_{n k}^{(1)} \phi_{k}^{(1)}+w_{n k}^{(1)}, \quad g_{n k}^{(1)} \phi_{k}^{(1)}=\square_{k} g_{n k}^{(1)} \phi_{k}^{(1)}, \quad \phi_{k}^{(1)} \in H^{1}
$$

and $(k, 1) \in \Lambda_{1,0} \cup \Lambda_{\infty, 1}$. Denote $u_{n}^{(1)}=u_{n}-g_{n k}^{(1)} \phi_{k}^{(1)}$ and suppose

$$
\square_{k} u_{n}^{(1)}=g_{n k}^{(2)} \phi_{k}^{(2)}+w_{n k}^{(2)}
$$

Repeating the proof above, we can get $g_{n k}^{(2)} \phi_{k}^{(2)}=\square_{k} g_{n k}^{(2)} \phi_{k}^{(2)}$, $\phi_{k}^{(2)} \in H^{1}$, and $(k, 2) \in \Lambda_{1,0} \cup \Lambda_{\infty, 1} \ldots$; by induction, we obtain (62).

By the orthogonal condition (45), following the proof in [14], we can obtain that for fix $k$ and for all $j \neq j^{\prime}$,

$$
\begin{aligned}
& \lim _{n \rightarrow \infty}\left\langle g_{n k}^{(j)} \phi_{k}^{(j)}, g_{n k}^{\left(j^{\prime}\right)} \phi_{k}^{\left(j^{\prime}\right)}\right\rangle_{\dot{H}^{1}\left(\mathbb{R}^{2}\right)} \\
& =\lim _{n \rightarrow \infty}\left\langle\square_{k} g_{n k}^{(j)} \phi_{k}^{(j)}, \square_{k} g_{n k}^{\left(j^{\prime}\right)} \phi_{k}^{\left(j^{\prime}\right)}\right\rangle_{\dot{H}^{1}\left(\mathbb{R}^{2}\right)}=0, \\
& \lim _{n \rightarrow \infty}\left\langle g_{n k}^{(j)} \phi_{k}^{(j)}, w_{n k}^{\left(l_{k}\right)}\right\rangle_{\dot{H}^{1}\left(\mathbb{R}^{2}\right)} \\
& =\lim _{n \rightarrow \infty}\left\langle\square_{k} g_{n k}^{(j)} \phi_{k}^{(j)}, \square_{k} w_{n k}^{\left(l_{k}\right)}\right\rangle_{\dot{H}^{1}\left(\mathbb{R}^{2}\right)}=0,
\end{aligned}
$$

(63) were proved. 
Step 2. For arbitrary $\left(k_{1}, j_{1}\right),\left(k_{2}, j_{2}\right) \in \Lambda_{1,0} \cup \Lambda_{\infty, 1}$, we define $\left(k_{1}, j_{1}\right) \sim\left(k_{2}, j_{2}\right)$ if the orthogonal condition (45) is NOT true for any subsequence; that is,

$$
\begin{aligned}
\limsup _{n \rightarrow \infty}( & \frac{\lambda_{n k_{1}}^{\left(j_{1}\right)}}{\lambda_{n k_{2}}^{\left(j_{2}\right)}}+\frac{\lambda_{n k_{2}}^{\left(j_{2}\right)}}{\lambda_{n k_{1}}^{\left(j_{1}\right)}}+\lambda_{n k_{2}}^{\left(j_{2}\right)} \lambda_{n k_{1}}^{\left(j_{1}\right)}\left|\xi_{n k_{2}}^{\left(j_{2}\right)}-\xi_{n k_{1}}^{\left(j_{1}\right)}\right|^{2} \\
& \left.+\frac{\left|x_{n k_{2}}^{\left(j_{2}\right)}-x_{n k_{1}}^{\left(j_{1}\right)}\right|^{2}}{\lambda_{n k_{2}}^{\left(j_{2}\right)} \lambda_{n k_{1}}^{\left(j_{1}\right)}}+\frac{\left|t_{n k_{2}}^{\left(j_{2}\right)}\left(\lambda_{n k_{2}}^{\left(j_{2}\right)}\right)^{2}-t_{n k_{1}}^{\left(j_{1}\right)}\left(\lambda_{n k_{1}}^{\left(j_{1}\right)}\right)^{2}\right|}{\lambda_{n k_{2}}^{\left(j_{2}\right)} \lambda_{n k_{1}}^{\left(j_{1}\right)}}\right)
\end{aligned}
$$

$<\infty$.

By the definition above, if $\left(k_{1}, j_{1}\right) \sim\left(k_{2}, j_{2}\right)$, we have

$$
\begin{gathered}
\lambda_{n k_{1}}^{\left(j_{1}\right)} \sim \lambda_{n k_{2}}^{\left(j_{2}\right)}, \quad \lambda_{n k_{1}}^{\left(j_{1}\right)} \xi_{n k_{1}}^{\left(j_{1}\right)} \sim \lambda_{n k_{2}}^{\left(j_{2}\right)} \xi_{n k_{2}}^{\left(j_{2}\right)}, \\
\frac{x_{n k_{1}}^{\left(j_{1}\right)}}{\lambda_{n k_{1}}^{\left(j_{1}\right)}} \sim \frac{x_{n k_{2}}^{\left(j_{2}\right)},}{\lambda_{n k_{2}}^{\left(j_{2}\right)}}, \quad t_{n k_{1}}^{\left(j_{1}\right)} \sim t_{n k_{2}}^{\left(j_{2}\right)} .
\end{gathered}
$$

Note that

$$
g_{\theta, \xi_{0}, x_{0}, \lambda, t_{0}} f(x):=\frac{1}{\lambda} e^{i \theta} e^{i(x / \lambda) \cdot \lambda \xi_{0}}\left(e^{i t_{0} \Delta} f\right)\left(\frac{x}{\lambda}-\frac{x_{0}}{\lambda}\right) .
$$

By Remark 15, we can put these two profiles together as one profile. Then, by denoting $\left(\Lambda_{1,0} \cup \Lambda_{\infty, 1}\right) / \sim$ as $\{1,2, \ldots, J\}$, we can obtain the sequence $\phi_{\alpha}^{J}, \alpha=1,2, \ldots, J$; and (52)-(56) were proved.

Specially, since $\phi_{k}^{(j)} \in H^{1}$ for each $(k, j) \in \Lambda_{1,0} \cup \Lambda_{\infty, 1}$, we have

$$
\sum_{(k, j) \in \Lambda_{1,0} \cup \Lambda_{\infty, 1}} \phi_{k}^{(j)} \in H^{1}
$$

for fixed $N$ and $l_{k}$, and hence $\phi_{\alpha}^{J} \in H^{1}\left(\mathbb{R}^{2}\right)$ for any fixed $J$ and $1 \leq \alpha \leq J$.

Step 3. We prove (57) now. By (56), we only need to prove that for all $m \in \mathbb{N}, m \geq 2$,

$$
\begin{gathered}
\lim _{J \rightarrow \infty} \limsup _{n \rightarrow \infty}\left\{\left\|u_{n}\right\|_{L^{2 m}\left(\mathbb{R}^{2}\right)}^{2 m}-\sum_{\alpha=1}^{J}\left\|g_{n \alpha} \phi_{\alpha}^{J}\right\|_{L^{2 m}\left(\mathbb{R}^{2}\right)}^{2 m}\right. \\
\left.-\|R(n, J)\|_{L^{2 m}\left(\mathbb{R}^{2}\right)}^{2 m}\right\}=0 .
\end{gathered}
$$

As

$$
\|R(n, J)\|_{L^{2 m}\left(\mathbb{R}^{2}\right)} \leqslant\|R(n, J)\|_{L^{4}\left(\mathbb{R}^{2}\right)}^{1 /(m-1)}\|R(n, J)\|_{H^{1}\left(\mathbb{R}^{2}\right)}^{(m-2) /(m-1)}
$$

and for $1 / 4<\theta<1 / 2$,

$$
\begin{aligned}
\| R & (n, J) \|_{L^{4}\left(\mathbb{R}^{2}\right)} \\
& \leq\left\|e^{i t \Delta} R(n, J)\right\|_{L_{t}^{\infty} L_{x}^{4}\left(\mathbb{R} \times \mathbb{R}^{2}\right)} \\
& \leq\left\|e^{i t \Delta} R(n, J)\right\|_{H_{4}^{\theta} L_{x}^{4}\left(\mathbb{R} \times \mathbb{R}^{2}\right)} \\
& =\left\|e^{i t \Delta} R(n, J)\right\|_{L_{t, x}^{4}\left(\mathbb{R} \times \mathbb{R}^{2}\right)}^{1-2 \theta}\left\|e^{i t \Delta} R(n, J)\right\|_{H_{4}^{1 / 2} L_{x}^{4}\left(\mathbb{R} \times \mathbb{R}^{2}\right)}^{2 \theta} \\
& =\left\|e^{i t \Delta} R(n, J)\right\|_{L_{t, x}^{4}\left(\mathbb{R} \times \mathbb{R}^{2}\right)}^{1-2 \theta}\left\|e^{i t \Delta} R(n, J)\right\|_{L_{t}^{4} H_{4}^{1}\left(\mathbb{R} \times \mathbb{R}^{2}\right)}^{2 \theta} \\
& \leq\left\|e^{i t \Delta} R(n, J)\right\|_{L_{t, x}^{4}\left(\mathbb{R} \times \mathbb{R}^{2}\right)^{.}}^{1-2 \theta}
\end{aligned}
$$

By (54), we have

$$
\lim _{J \rightarrow \infty} \limsup _{n \rightarrow \infty}\|R(n, J)\|_{L^{2 m}\left(\mathbb{R}^{2}\right)}^{2 m}=0 .
$$

We separate the set $1 \leq \alpha \leq J$ into two subsets:

$$
\begin{gathered}
\Lambda_{1}=\left\{\alpha \mid 1 \leq \alpha \leq J, \lambda_{n \alpha} \equiv 1, \xi_{n \alpha} \equiv 0\right\}, \\
\Lambda_{\infty}=\left\{\alpha \mid 1 \leq \alpha \leq J, \lambda_{n \alpha} \longrightarrow \infty\right\} .
\end{gathered}
$$

When $\alpha \in \Lambda_{\infty}$,

$$
\begin{aligned}
\lim _{n \rightarrow \infty}\left\|g_{n \alpha} \phi_{\alpha}^{J}\right\|_{L^{2 m}\left(\mathbb{R}^{2}\right)} & =\lim _{n \rightarrow \infty}\left(\lambda_{n \alpha}\right)^{-1+1 / m}\left\|e^{i t_{n \alpha} \Delta} \phi_{\alpha}^{J}\right\|_{L^{2 m}\left(\mathbb{R}^{2}\right)} \\
& \leq \lim _{n \rightarrow \infty}\left(\lambda_{n \alpha}\right)^{-1+1 / m}\left\|\phi_{\alpha}^{J}\right\|_{H^{1}\left(\mathbb{R}^{2}\right)}=0 .
\end{aligned}
$$

Hence, in order to prove (90), one only needs to prove

$$
\lim _{n \rightarrow \infty}\left\{\left\|\sum_{\alpha \in \Lambda_{1}} g_{n \alpha} \phi_{\alpha}^{J}\right\|_{L^{2 m}\left(\mathbb{R}^{2}\right)}^{2 m}-\sum_{\alpha \in \Lambda_{1}}\left\|g_{n \alpha} \phi_{\alpha}^{J}\right\|_{L^{2 m}\left(\mathbb{R}^{2}\right)}^{2 m}\right\}=0 .
$$

If $\alpha \in \Lambda_{1}$ and $t_{n \alpha} \rightarrow \infty$, for a function $\widetilde{\phi}_{\alpha}^{J} \in \dot{H}^{1 / 2} \cap L^{4 / 3}$, we have

$$
\begin{aligned}
\left\|g_{n \alpha} \phi_{\alpha}^{J}\right\|_{L^{4}\left(\mathbb{R}^{2}\right)} & \leq\left\|g_{n \alpha} \phi_{\alpha}^{J}-g_{n \alpha} \widetilde{\phi}_{\alpha}^{J}\right\|_{L^{4}\left(\mathbb{R}^{2}\right)}+\left\|g_{n \alpha} \widetilde{\phi}_{\alpha}^{J}\right\|_{L^{4}\left(\mathbb{R}^{2}\right)} \\
& \leq\left\|\phi_{\alpha}^{J}-\widetilde{\phi}_{\alpha}^{J}\right\|_{\dot{H}^{1 / 2}\left(\mathbb{R}^{2}\right)}+\left|t_{n \alpha}\right|^{-1 / 2}\left\|\widetilde{\phi}_{\alpha}^{J}\right\|_{L^{4 / 3}\left(\mathbb{R}^{2}\right)} .
\end{aligned}
$$

By approximating $\phi_{\alpha}^{J}$ by $\widetilde{\phi}_{\alpha}^{J} \in C_{c}^{\infty}$ in $\dot{H}^{1 / 2}$ and sending $n \rightarrow$ $\infty$, we have $\left\|g_{n \alpha} \phi_{\alpha}^{J}\right\|_{L^{4}\left(\mathbb{R}^{2}\right)} \rightarrow 0$. Note that $g_{n \alpha} \phi_{\alpha}^{J} \in H^{1}$; we obtain $\left\|g_{n \alpha} \phi_{\alpha}^{J}\right\|_{L^{2 m}\left(\mathbb{R}^{2}\right)} \rightarrow 0$ for all $m \geq 2$. 
If $\alpha \in \Lambda_{1}$ and $t_{n \alpha} \equiv 0$, we have orthogonal condition $\left|x_{n \alpha}-x_{n \alpha^{\prime}}\right| \rightarrow \infty$ for any $\alpha \neq \alpha^{\prime}$. Thus,

$$
\begin{gathered}
\lim _{n \rightarrow \infty}\left\{\left\|\sum_{\alpha \in \Lambda_{1}, t_{n \alpha} \equiv 0} g_{n \alpha} \phi_{\alpha}^{J}\right\|_{L^{2 m}\left(\mathbb{R}^{2}\right)}^{2 m}-\sum_{\alpha \in \Lambda_{1}, t_{n \alpha} \equiv 0}\left\|g_{n \alpha} \phi_{\alpha}^{J}\right\|_{L^{2 m}\left(\mathbb{R}^{2}\right)}^{2 m}\right\} \\
=\lim _{n \rightarrow \infty}\left\{\left\|\sum_{\alpha \in \Lambda_{1}, t_{n \alpha} \equiv 0} e^{i \theta_{n \alpha}} \phi_{\alpha}^{J}\left(\cdot-x_{n \alpha}\right)\right\|_{L^{2 m}\left(\mathbb{R}^{2}\right)}^{2 m}\right. \\
\left.\quad-\sum_{\alpha \in \Lambda_{1}, t_{n \alpha} \equiv 0}\left\|\phi_{\alpha}^{J}\left(\cdot-x_{n \alpha}\right)\right\|_{L^{2 m}\left(\mathbb{R}^{2}\right)}^{2 m}\right\}=0 .
\end{gathered}
$$

(96) holds and then (57) was proved.

\section{The Proof of Theorem 3}

Let $u$ be a solution of (5), $H\left(u_{0}\right) \leq 1$; by Strichartz estimate and (21),

$$
\begin{aligned}
& \|u\|_{L_{t, x}^{4} \times L_{t}^{2 /(1-2 \varepsilon)} L_{x}^{1 / \varepsilon}\left(\mathbb{R} \times \mathbb{R}^{2}\right)} \\
& \leq C\left\|u_{0}\right\|_{L^{2}\left(\mathbb{R}^{2}\right)}+C\|u\|_{L_{t, x}^{4} \times\left(\mathbb{R} \times \mathbb{R}^{2}\right)}^{3} \\
& \quad+C(E)\|u\|_{L_{t}^{2 / 1-2 \varepsilon)} L_{x}^{1 / \varepsilon}\left(\mathbb{R} \times \mathbb{R}^{2}\right)}\|u\|_{L_{t, x}^{4}\left(\mathbb{R} \times \mathbb{R}^{2}\right)}^{8 \varepsilon} \\
& \leq C\left\|u_{0}\right\|_{L^{2}\left(\mathbb{R}^{2}\right)}+C\|u\|_{L_{t, x}^{4} \cap L_{t}^{2 /(1-2 \varepsilon)} L_{x}^{1 / \varepsilon}\left(\mathbb{R} \times \mathbb{R}^{2}\right)}^{3} \\
& \quad+C(E)\|u\|_{L_{t, x}^{4} \cap L_{t}^{2 /(1-2 \varepsilon)} L_{x}^{1 / \varepsilon}\left(\mathbb{R} \times \mathbb{R}^{2}\right)^{1} .}^{1+8 \varepsilon}
\end{aligned}
$$

When $\left\|u_{0}\right\|_{L^{2}\left(\mathbb{R}^{2}\right)} \ll 1$, by standard continuity argument, we have

$$
\|u\|_{L_{t, x}^{4}\left(\mathbb{R} \times \mathbb{R}^{2}\right)} \leq C\left\|u_{0}\right\|_{L^{2}\left(\mathbb{R}^{2}\right)}<\infty .
$$

Hence, if $M(u) \ll 1$, then $\|u\|_{L_{t, x}^{4}\left(\mathbb{R} \times \mathbb{R}^{2}\right)}<\infty$. In particular, we have scattering in both directions.

For any mass $m \geq 0$, we define

$A(m):=\sup \{S(u): u$ is the global solution of (5),

$$
M(u) \leq m, H(u) \leq 1\} .
$$

Then $A:[0,+\infty) \rightarrow[0,+\infty]$ is a monotone increasing function of $m$. As $A$ is left-continuous and finite for small $m$, there must exist a unique critical mass $m_{0} \in(0+\infty)$ such that $A(m)$ is finite for all $m<m_{0}$ but infinite for all $m \geq m_{0}$.

To prove Theorem 3 , one only needs to prove that the critical mass $m_{0}$ is infinite. We will prove that by contradiction.

Proposition 17. Suppose that the critical mass $m_{0}$ is finite. Let $u_{n}: \mathbb{R} \times \mathbb{R}^{2} \rightarrow \mathbb{C}$ for $n=1,2, \ldots$ be a sequence of solutions and let $t_{n} \in \mathbb{R}$ be a sequence of times such that $H\left(u_{n}\right) \leq 1$, $\lim \sup _{n \rightarrow \infty} M\left(u_{n}\right)=m_{0}$, and

$$
\lim _{n \rightarrow \infty} S_{\geq t_{n}}\left(u_{n}\right)=\lim _{n \rightarrow \infty} S_{\leq t_{n}}\left(u_{n}\right)=+\infty .
$$

Then there exists a sequence of $x_{n}=x_{n}\left(t_{n}\right) \in \mathbb{R}^{2}$ such that $u_{n}\left(t_{n}, x+x_{n}\right)$ has a subsequence which converges strongly into $L_{x}^{2}\left(\mathbb{R}^{2}\right)$. Especially, the Hamiltonian of the limiting function is not greater than 1 .

Proof. We can take $t_{n}=0$ for all $n$ by translating $u_{n}$ in time. Thus,

$$
\lim _{n \rightarrow \infty} S_{\geq 0}\left(u_{n}\right)=\lim _{n \rightarrow \infty} S_{\leq 0}\left(u_{n}\right)=+\infty .
$$

By Lemma 16, up to a subsequence if necessary, we have

$$
u_{n}(0)=\sum_{\alpha \in \Lambda_{1} \cup \Lambda_{\infty}} g_{n \alpha} \phi_{\alpha}^{J}+R(n, J),
$$

where $\Lambda_{1}$ and $\Lambda_{\infty}$ were defined by (94). Suppose that

$$
g_{n \alpha}=h_{n \alpha} e^{i t_{n \alpha} \Delta},
$$

where $t_{n \alpha} \in \mathbb{R}$ and $h_{n \alpha} \in G$. By (55),

$$
\sum_{\alpha \in \Lambda_{1} \cup \Lambda_{\infty}} M\left(\phi_{\alpha}^{J}\right) \leq \limsup _{n \rightarrow \infty} M\left(u_{n}(0)\right) \leq m_{0} .
$$

Hence,

$$
\sup _{\alpha \in \Lambda_{1} \cup \Lambda_{\infty}} M\left(\phi_{\alpha}^{J}\right) \leq m_{0} .
$$

Suppose

$$
\sup _{\alpha \in \Lambda_{1} \cup \Lambda_{\infty}} M\left(\phi_{\alpha}^{J}\right) \leq m_{0}-\sigma .
$$

For some $\sigma>0$, we will prove that this leads to a contradiction: defining the nonlinear profile $v_{\alpha}^{J}: \mathbb{R} \times \mathbb{R}^{2} \rightarrow \mathbb{C}$ as follows.

(i) When $\alpha \in \Lambda_{1}$,

(1) if $t_{n \alpha} \equiv 0$, we define $v_{\alpha}^{J}$ to be the global solution of (5) with initial data $v_{\alpha}^{J}(0)=\phi_{\alpha}^{J}$;

(2) if $t_{n \alpha} \rightarrow+\infty$, we define $v_{\alpha}^{J}$ to be the global solution of (5) which scatters to $e^{i t \Delta} \phi_{\alpha}^{J}$ in $H^{1}$ when $t \rightarrow+\infty$

(3) if $t_{n \alpha} \rightarrow-\infty$, we define $v_{\alpha}^{J}$ to be the global solution of (5) which scatters to $e^{i t \Delta} \phi_{\alpha}^{J}$ in $H^{1}$ when $t \rightarrow-\infty$.

(ii) When $\alpha \in \Lambda_{\infty}$,

(1) if $t_{n \alpha} \equiv 0$, we define $v_{\alpha}^{J}$ to be the global solution of $i u_{t}+\Delta u=|u|^{2} u$ with initial data $v_{\alpha}^{J}(0)=\phi_{\alpha}^{J}$;

(2) if $t_{n \alpha} \rightarrow+\infty$, we define $v_{\alpha}^{J}$ to be the global solution of $i u_{t}+\Delta u=|u|^{2} u$ which scatters to $e^{i t \Delta} \phi_{\alpha}^{J}$ in $H^{1}$ when $t \rightarrow+\infty$;

(3) if $t_{n \alpha} \rightarrow-\infty$, we define $v_{\alpha}^{J}$ to be the global solution of $i u_{t}+\Delta \mathrm{u}=|u|^{2} u$ which scatters to $e^{i t \Delta} \phi_{\alpha}^{J}$ in $H^{1}$ when $t \rightarrow-\infty$. 
For each $\alpha \in \Lambda_{1}, v_{\alpha}^{J}$ is well defined by the definition of $A(m)$, (100), and (108). For each $\alpha \in \Lambda_{\infty}, v_{\alpha}^{J}$ is well defined by the scattering of cubic Schrödinger equation in $L^{2}\left(\mathbb{R}^{2}\right)$ (see $[7,8])$ and the same analysis as in Lemma 9.

Now, we define

$$
\widetilde{u}_{n}=\sum_{\alpha \in \Lambda_{1} \cup \Lambda_{\infty}} T_{h_{n \alpha}}\left[v_{\alpha}^{J}\left(\cdot+t_{n \alpha}\right)\right]+e^{i t \Delta} R(n, J)
$$

for $n, J=1,2, \ldots$, and then we have the following two lemmas.

\section{Lemma 18}

$$
\begin{aligned}
& \lim _{n \rightarrow \infty} M\left(\widetilde{u}_{n}(0)-u_{n}(0)\right)=0, \\
& \lim _{J \rightarrow \infty} \limsup _{n \rightarrow \infty}\left\|\widetilde{u}_{n}\right\|_{\dot{H}^{1}\left(\mathbb{R}^{2}\right)}^{2} \leq 1 .
\end{aligned}
$$

Proof. Since

$$
\begin{aligned}
\lim _{n \rightarrow \infty} M\left(\widetilde{u}_{n}(0)-u_{n}(0)\right) \\
\quad \leq \sum_{\alpha \in \Lambda_{1} \cup \Lambda_{\infty}} \lim _{n \rightarrow \infty} M\left(T_{h_{n \alpha}}\left[v_{\alpha}^{J}\left(\cdot+t_{n \alpha}\right)\right](0)-g_{n \alpha} \phi_{\alpha}^{J}\right) \\
=\sum_{\alpha \in \Lambda_{1} \cup \Lambda_{\infty}} \lim _{n \rightarrow \infty} M\left(h_{n \alpha}\left[v_{\alpha}^{J}\left(t_{n \alpha}\right)\right]-h_{n \alpha} e^{i t_{n \alpha} \Delta} \phi_{\alpha}^{J}\right) \\
=\sum_{\alpha \in \Lambda_{1} \cup \Lambda_{\infty}} \lim _{n \rightarrow \infty} M\left(v_{\alpha}^{J}\left(t_{n \alpha}\right)-e^{i t_{n \alpha} \Delta} \phi_{\alpha}^{J}\right)=0,
\end{aligned}
$$

we get the first equality.

Note that $T_{h_{n x}}$ maps the solutions of $i u_{t}+\Delta u=|u|^{2} u$ from one to another; using (57) and energy conservation, we have

$$
\begin{aligned}
& \lim _{J \rightarrow \infty} \limsup _{n \rightarrow \infty}\left\|\widetilde{u}_{n}\right\|_{\dot{H}^{1}\left(\mathbb{R}^{2}\right)}^{2} \\
& \leq \lim _{J \rightarrow \infty} \limsup _{n \rightarrow \infty}\left\{\sum_{\alpha=1}^{J}\left\|T_{h_{n \alpha}}\left[v_{\alpha}^{J}\left(\cdot+t_{n \alpha}\right)\right]\right\|_{H^{1}\left(\mathbb{R}^{2}\right)}^{2}\right. \\
& \left.+\|R(n, J)\|_{\dot{H}^{1}\left(\mathbb{R}^{2}\right)}^{2}\right\} \\
& \leq \lim _{J \rightarrow \infty} \limsup _{n \rightarrow \infty}\left\{\sum_{\alpha=1}^{J} H\left(g_{n \alpha} \phi_{\alpha}^{J}\right)+H(R(n, J))\right\} \\
& \leq \lim _{J \rightarrow \infty} \limsup _{n \rightarrow \infty} H\left(u_{n}\right) \leq 1 .
\end{aligned}
$$

Lemma 19. If

$$
\begin{gathered}
\lim _{J \rightarrow \infty} \limsup _{n \rightarrow \infty}\left\|\tilde{u}_{n}\right\|_{L_{t, x}^{4}\left(\mathbb{R} \times \mathbb{R}^{2}\right)}<\infty, \\
\left\|v_{\alpha}^{J}\right\|_{L_{t, x}^{4}\left(\mathbb{R} \times \mathbb{R}^{2}\right)}<\infty \quad(\forall \alpha),
\end{gathered}
$$

then

$\lim _{J \rightarrow \infty} \limsup _{n \rightarrow \infty}\left\|\int_{0}^{t} e^{i(t-\tau) \Delta}\left(\left(i \partial_{t}+\Delta\right) \tilde{u}_{n}-f\left(\widetilde{u}_{n}\right)\right)(\tau) d \tau\right\|_{X}=0$,

where $X=L_{t, x}^{4} \cap L_{t}^{2 /(1-2 \varepsilon)} L_{x}^{1 / \varepsilon}\left(\mathbb{R} \times \mathbb{R}^{2}\right)$.
Proof. Denote

$$
v_{n \alpha}^{J}=T_{h_{n \alpha}}\left[v_{\alpha}^{J}\left(\cdot+t_{n \alpha}\right)\right] .
$$

By the definition of $\widetilde{u}_{n}$, we have

$$
\widetilde{u}_{n}=\sum_{\alpha \in \Lambda_{1} \cup \Lambda_{\infty}} v_{n \alpha}^{J}+e^{i t \Delta} R(n, J),
$$

$$
\left(i \partial_{t}+\Delta\right) \widetilde{u}_{n}=\sum_{\alpha \in \Lambda_{1}} f\left(v_{n \alpha}^{J}\right)+\sum_{\alpha \in \Lambda_{\infty}}\left|v_{n \alpha}^{J}\right|^{2} v_{n \alpha}^{J} .
$$

Thus, by triangle inequality, it suffices to show that

$$
\begin{aligned}
\lim _{J \rightarrow \infty} \limsup _{n \rightarrow \infty} \| \int_{0}^{t} e^{i(t-\tau) \Delta}( & f\left(\widetilde{u}_{n}-e^{i t \Delta} R(n, J)\right) \\
& \left.-f\left(\widetilde{u}_{n}\right)\right)(\tau) d \tau \|_{X}=0,
\end{aligned}
$$

$$
\begin{aligned}
& \lim _{n \rightarrow \infty}\left\|\int_{0}^{t} e^{i(t-\tau) \Delta} \sum_{\alpha \in \Lambda_{\infty}}\left(f\left(v_{n \alpha}^{J}\right)-\left|v_{n \alpha}^{J}\right|^{2} v_{n \alpha}^{J}\right)(\tau) d \tau\right\|_{X} \\
& \quad=0 .
\end{aligned}
$$

By Lemma 18, let $J$ and $n$ be sufficiently large; we have

$$
\left\|\widetilde{u}_{n}\right\|_{\dot{H}^{1}\left(\mathbb{R}^{2}\right)}^{2} \leq \frac{1}{1-4 \varepsilon}, \quad\left\|\widetilde{u}_{n}-e^{i t \Delta} R(n, J)\right\|_{\dot{H}^{1}\left(\mathbb{R}^{2}\right)}^{2} \leq \frac{1}{1-4 \varepsilon} .
$$

Since we have supposed $0<\lambda<4(1-4 \varepsilon)^{2} \pi$, Lemma 6 can also be used here. Then by the same estimates as (31),

$$
\begin{aligned}
& \left\|\int_{0}^{t} e^{i(t-\tau) \Delta}\left(f\left(\widetilde{u}_{n}-e^{i t \Delta} R(n, J)\right)-f\left(\widetilde{u}_{n}\right)\right)(\tau) d \tau\right\|_{X} \\
& \leq\left(\left\|\widetilde{u}_{n}\right\|_{L_{t, x}^{4}\left(\mathbb{R} \times \mathbb{R}^{2}\right)}^{2}+\left\|e^{i t \Delta} R(n, J)\right\|_{L_{t, x}^{4}\left(\mathbb{R} \times \mathbb{R}^{2}\right)}^{2}\right) \\
& \quad \times\left\|e^{i t \Delta} R(n, J)\right\|_{L_{t, x}^{4}\left(\mathbb{R} \times \mathbb{R}^{2}\right)} \\
& \quad+\left(\left\|\widetilde{u}_{n}\right\|_{L_{t, x}^{4}\left(\mathbb{R} \times \mathbb{R}^{2}\right)}^{8 \varepsilon}+\left\|e^{i t \Delta} R(n, J)\right\|_{L_{t, x}^{4}\left(\mathbb{R} \times \mathbb{R}^{2}\right)}^{8 \varepsilon}\right) \\
& \quad \times\left\|e^{i t \triangle} R(n, J)\right\|_{L_{t}^{2 /(1-2 \varepsilon)} L_{x}^{1 / \varepsilon}\left(\mathbb{R} \times \mathbb{R}^{2}\right)^{2}}
\end{aligned}
$$

By (54),

$$
\lim _{J \rightarrow \infty} \limsup _{n \rightarrow \infty}\left\|e^{i t \Delta} R(n, J)\right\|_{L_{t, x}^{4}\left(\mathbb{R} \times \mathbb{R}^{2}\right)}=0 .
$$


As

$$
\begin{aligned}
& \left\|e^{i t \Delta} R(n, J)\right\|_{L_{t}^{2 /(1-2 \varepsilon)} L_{x}^{1 / \varepsilon}\left(\mathbb{R} \times \mathbb{R}^{2}\right)} \\
& \quad \leqslant\left\|e^{i t \Delta} R(n, J)\right\|_{L_{t, x}^{4}\left(\mathbb{R} \times \mathbb{R}^{2}\right)}\left\|e^{i t \Delta} R(n, J)\right\|_{L_{t}^{2 /(1-\varepsilon)} L_{x}^{2 / \varepsilon}\left(\mathbb{R} \times \mathbb{R}^{2}\right)^{\prime}}^{(1-4 \varepsilon) /(1-2 \varepsilon)} \\
& \left\|e^{i t \Delta} R(n, J)\right\|_{L_{t}^{2 /(1-\varepsilon)} L_{x}^{2 / \varepsilon}\left(\mathbb{R} \times \mathbb{R}^{2}\right)} \leq\|R(n, J)\|_{L_{x}^{2}\left(\mathbb{R}^{2}\right)}<\infty,
\end{aligned}
$$

we have

$$
\lim _{J \rightarrow \infty} \limsup _{n \rightarrow \infty}\left\|e^{i t \Delta} R(n, J)\right\|_{L_{t}^{2 /(1-2 \varepsilon)} L_{x}^{1 / \varepsilon}\left(\mathbb{R} \times \mathbb{R}^{2}\right)}=0 .
$$

By $\lim _{J \rightarrow \infty} \lim \sup _{n \rightarrow \infty}\left\|\tilde{u}_{n}\right\|_{L_{t, x}^{4}\left(\mathbb{R} \times \mathbb{R}^{2}\right)}<\infty$, (117) was obtained.

Using Strichartz estimate,

$$
\begin{aligned}
& \left\|\int_{0}^{t} e^{i(t-\tau) \Delta}\left(f\left(v_{n \alpha}^{J}\right)-\left|v_{n \alpha}^{J}\right|^{2} v_{n \alpha}^{J}\right)(\tau) d \tau\right\|_{X} \\
& \quad \leq\left\|\sum_{m=2}^{\infty} \frac{\left|v_{n \alpha}^{J}\right|^{2 m} v_{n \alpha}^{J}}{m !}\right\|_{L_{t, x}^{4 / 3}\left(\mathbb{R} \times \mathbb{R}^{2}\right)} \\
& \quad \leq \sum_{m=2}^{\infty} \frac{1}{m !}\left\|v_{n \alpha}^{J}\right\|_{L_{t, x}^{4}\left(\mathbb{R} \times \mathbb{R}^{2}\right)}\left\|v_{n \alpha}^{J}\right\|_{L_{t, x}^{4 m}\left(\mathbb{R} \times \mathbb{R}^{2}\right)}^{2 m} \\
& \quad \leq \sum_{m=2}^{\infty} \frac{1}{m !}\left(\lambda_{n \alpha}\right)^{-2(m-1)}\left\|v_{\alpha}^{J}\right\|_{L_{t, x}^{4}\left(\mathbb{R} \times \mathbb{R}^{2}\right)}\left\|v_{\alpha}^{J}\right\|_{L_{t, x}^{4 m}\left(\mathbb{R} \times \mathbb{R}^{2}\right)}^{2 m} .
\end{aligned}
$$

By Lemma 8 ,

$$
\left\|v_{\alpha}^{J}\right\|_{L_{t, x}^{4 m}\left(\mathbb{R} \times \mathbb{R}^{2}\right)} \leq C m^{1 / 2+1 / 2 m}\left\|v_{\alpha}^{J}\right\|_{L_{t, x}^{4}\left(\mathbb{R} \times \mathbb{R}^{2}\right)}^{1 / m}\left\|v_{\alpha}^{J}\right\|_{L^{\infty}\left(\mathbb{R}, \dot{H}^{1}\right)}^{1-1 / m} .
$$

Note that $\left\|v_{\alpha}^{J}\right\|_{L_{t, x}^{4}\left(\mathbb{R} \times \mathbb{R}^{2}\right)}<\infty$. Equation (119) was obtained.

To prove Lemma 19 , it is only left to prove (118). Note that

$$
\left|f\left(\sum_{\alpha=1}^{J} z_{\alpha}\right)-\sum_{\alpha=1}^{J} f\left(z_{\alpha}\right)\right| \lesssim \sum_{\alpha \neq \alpha^{\prime}}\left|z_{\alpha}\right|\left|e^{\lambda\left|z_{\alpha^{\prime}}\right|^{2}}-1\right| .
$$

By (57), we have

$$
\left\|\int_{0}^{t} e^{i(t-\tau) \Delta}\left(f\left(\sum_{\alpha \in \Lambda_{1} \cup \Lambda_{\infty}} v_{n \alpha}^{J}\right)-\sum_{\alpha \in \Lambda_{1} \cup \Lambda_{\infty}} f\left(v_{n \alpha}^{J}\right)\right)(\tau) d \tau\right\|_{X}
$$

$$
\begin{aligned}
& \lesssim \sum_{\alpha \neq \alpha^{\prime}}\left(\left\|v_{n \alpha}^{J}\left|v_{n \alpha^{\prime}}^{J}\right|^{2}\right\|_{L_{t, \alpha}^{4 / 3}\left(\mathbb{R} \times \mathbb{R}^{2}\right)}\right. \\
& \left.+\left\|v_{n \alpha}^{J}\left(e^{\lambda\left|v_{n \alpha^{\prime}}^{J}\right|^{2}}-\lambda\left|v_{n \alpha^{\prime}}^{J}\right|^{2}-1\right)\right\|_{L_{t}^{2 /(1+2 \varepsilon)} L_{x}^{1 /(1-\varepsilon)}\left(\mathbb{R} \times \mathbb{R}^{2}\right)}\right) \\
& \lesssim \sum_{\alpha \neq \alpha^{\prime}}\left\|\left|v_{n \alpha}^{J}\right|^{1 / 2}\left|v_{n \alpha^{\prime}}^{J}\right|^{1 / 2}\right\|_{L_{t, x}^{4}\left(\mathbb{R} \times \mathbb{R}^{2}\right)}^{2}\left\|v_{n \alpha^{\prime}}^{J}\right\|_{L_{t, x}^{4}\left(\mathbb{R} \times \mathbb{R}^{2}\right)} \\
& +\sum_{\alpha \neq \alpha^{\prime}}\left\|\left|v_{n \alpha}^{J}\right|^{\varepsilon /(1+\varepsilon)}\left|v_{n \alpha^{\prime}}^{J}\right|^{1 /(1+\varepsilon)}\right\|_{L_{t, x}^{4}\left(\mathbb{R} \times \mathbb{R}^{2}\right)}^{4 \varepsilon(1+\varepsilon) /(1-2 \varepsilon)}
\end{aligned}
$$

$$
\begin{aligned}
& \times\left\|v_{n \alpha}^{J}\right\|_{L_{t}^{2\left(1-2 \varepsilon-4 \varepsilon^{2}\right) /\left(1-2 \varepsilon-6 \varepsilon^{2}\right)} L_{x}^{\left(1-2 \varepsilon-4 \varepsilon^{2}\right) / \varepsilon^{2}}\left(\mathbb{R} \times \mathbb{R}^{2}\right)}^{\left(1-2 \varepsilon \varepsilon^{2}\right) /(1-2 \varepsilon)} \\
& \times \|\left|v_{n \alpha^{\prime}}^{J}\right|^{-4 \varepsilon /(1-2 \varepsilon)} \\
& \times\left(e^{\lambda \mid v_{n \alpha^{\prime}}^{J} \prime^{2}}-\lambda\left|v_{n \alpha^{\prime}}^{J}\right|^{2}-1\right) \|_{L_{t}^{\infty} L_{x}^{(1-2 \varepsilon) /(1-4 \varepsilon)}\left(\mathbb{R} \times \mathbb{R}^{2}\right)} .
\end{aligned}
$$

By (49), we immediately obtain (118).

If

$$
\sup _{\alpha \in \Lambda_{1} \cup \Lambda_{\infty}} M\left(\phi_{\alpha}\right) \leq m_{0}-\sigma
$$

for some $\sigma>0$, by the definition of $A(m)$ and (100), we have

$$
A(m) \leq B m \quad \forall m \in\left[0, m_{0}-\sigma\right],
$$

where $B=B(\sigma) \in(0,+\infty)$. Then $v_{\alpha}^{J}$ satisfies

$$
\begin{gathered}
M\left(v_{\alpha}^{J}\right)=M\left(\phi_{\alpha}^{J}\right) \leq m_{0}-\sigma, \\
S\left(v_{\alpha}^{J}\right) \leq A\left(M\left(\phi_{\alpha}^{J}\right)\right) \leq B M\left(\phi_{\alpha}^{J}\right) .
\end{gathered}
$$

By (50), (106), and (132), we have

$$
\lim _{J \rightarrow \infty} \limsup _{n \rightarrow \infty} S\left(\widetilde{u}_{n}\right) \leq B m_{0} .
$$

Using Lemmas 18 and 19, we have

$$
\begin{aligned}
& M\left(\widetilde{u}_{n}(0)-u_{n}(0)\right) \leq \delta, \quad S\left(\widetilde{u}_{n}\right) \leq 2 B m_{0}, \\
& \left\|\int_{0}^{t} e^{i(t-\tau) \Delta}\left(\left(i \partial_{t}+\Delta\right) \widetilde{u}_{n}-f\left(\widetilde{u}_{n}\right)\right)(\tau) d \tau\right\|_{L_{t, x}^{4} \times L_{t}^{2 /(1-2 \varepsilon)} L_{x}^{1 / \varepsilon}\left(\mathbb{R} \times \mathbb{R}^{2}\right)} \\
& \quad \leq \delta
\end{aligned}
$$

for $\delta>0$ sufficiently small, $J=J(\delta)$, and $n=n(J, \delta)$ sufficiently large. By Lemma 10 , we obtain that $S\left(u_{n}\right) \leq 3 B m_{0}$ which contradicts (103). Thus, (129) fails for all $\sigma>0$, and then

$$
\sup _{\alpha \in \Lambda_{1} \cup \Lambda_{\infty}} M\left(\phi_{\alpha}\right)=m_{0} .
$$

Comparing this with (106), we have

$$
u_{n}(0)=h_{n} e^{i t_{n} \Delta} \phi+R_{n}
$$


with $t_{n}$ converging to $\pm \infty$ or $t_{n} \equiv 0, h_{n} \in G, M(\phi)=m_{0}$, and

$$
M\left(R_{n}\right) \longrightarrow 0, \quad S\left(e^{i t \Delta} R_{n}\right) \longrightarrow 0 \quad \text { as } n \longrightarrow \infty .
$$

Specially, the parameters $\lambda_{n}, \xi_{n}$ of $h_{n}$ must satisfy

$$
\text { “ } \lambda_{n} \equiv 1 \text { and } \xi_{n} \equiv 0 \text {," or " } \lambda_{n} \longrightarrow \infty \text {." }
$$

If $\lambda_{n} \rightarrow \infty$, similar to the former case, we can define the approximate solution

$$
\tilde{u}_{n}=T_{h_{n}}\left[v\left(\cdot+t_{n}\right)\right]+e^{i t \Delta} R_{n} .
$$

By the scattering of cubic Schrödinger equation, we have $S(v)<\infty$ and $\lim _{n \rightarrow \infty} S\left(\tilde{u}_{n}\right)<\infty$. By Lemmas 18, 19, and 10 , for $n$ sufficiently large, we obtain $S\left(u_{n}\right)<\infty$ which contradicts (103).

If $\lambda_{n} \equiv 1, \xi_{n} \equiv 0$, and $t_{n} \rightarrow+\infty$, by Strichartz estimate and monotone convergence, we have

$$
\lim _{n \rightarrow \infty} S_{\geq 0}\left(e^{i t \Delta} e^{i t_{n} \Delta} \phi\right)=0
$$

Thus,

$$
\lim _{n \rightarrow \infty} S_{\geq 0}\left(e^{i t \Delta} h_{n} e^{i t_{n} \Delta} \phi\right)=\lim _{n \rightarrow \infty} S_{\geq 0}\left(e^{i t \Delta} e^{i t_{n} \Delta} \phi\right)=0 .
$$

Since $\lim _{n \rightarrow \infty} S\left(e^{i t \triangle} R_{n}\right)=0$, we can see from (136) that

$$
\lim _{n \rightarrow \infty} S_{\geq 0}\left(e^{i t \Delta} u_{n}(0)\right)=0 .
$$

Hence,

$$
\begin{aligned}
& \lim _{n \rightarrow \infty}\left\|\int_{0}^{t} e^{i(t-\tau) \Delta} f\left(e^{i t \Delta} u_{n}(0)\right)(\tau) d \tau\right\|_{L_{t, x}^{4} \cap L_{t}^{2 /(1-2 \varepsilon)} L_{x}^{1 / \varepsilon}\left([0, \infty) \times \mathbb{R}^{2}\right)} \\
& \quad=0 .
\end{aligned}
$$

By Lemma 10 (with $e^{i t \triangle} u_{n}(0)$ as the approximate solution and $u_{n}(0)$ as the initial data), we have

$$
\lim _{n \rightarrow \infty} S_{\geq 0}\left(u_{n}\right)=0,
$$

which contradicts one of the estimates in (103).

If $\lambda_{n} \equiv 1, \xi_{n} \equiv 0$, and $t_{n} \rightarrow-\infty$, the argument is the same and we can obtain a contradiction by using the other half of (103).

Now, the only case left is $\lambda_{n} \equiv 1, \xi_{n} \equiv 0$, and $t_{n} \equiv 0$. In this case, we have

$$
M\left(u_{n}(0)-h_{n} \phi\right)=M\left(R_{n}\right) \longrightarrow 0 \quad \text { as } n \longrightarrow \infty \text {. }
$$

Thus, $\left(h_{n}\right)^{-1} u_{n}(0)=e^{i \theta_{n}} u_{n}\left(0, x+x_{n}\right)$ converges to $\phi$ in $L_{x}^{2}\left(\mathbb{R}^{2}\right)$. Since $H(\phi)=H\left(g_{n} \phi\right) \leq 1$, after passing to a subsequence if necessary and rotating $\phi$, the desired result follows.

Let $\left\{u_{n}\right\}$ be the sequence given in Proposition 17 and satisfy $M\left(u_{n}\right) \leq m_{0}$ and suppose that $u_{n}\left(0, x+x_{n}\right)$ converges to $u_{0}$ strongly in $L_{x}^{2}\left(\mathbb{R}^{2}\right)$; then $M\left(u_{0}\right) \leq m_{0}$ and $H\left(u_{0}\right) \leq 1$.
Let $u$ be the global solution with initial data $u(0)=u_{0}$; by Lemma 10, we must have

$$
S_{\geq 0}(u)=S_{\leq 0}(u)=+\infty .
$$

By the definition of $m_{0}, M\left(u_{0}\right) \geq m_{0}$ and hence $M\left(u_{0}\right)=m_{0}$.

Since $u$ is locally in $L_{t, x}^{4}$, for all $t_{n} \in \mathbb{R}$, we have

$$
S_{\geq t_{n}}(u)=S_{\leq t_{n}}(u)=+\infty
$$

Using Proposition 17 for $\left\{u\left(t_{n}\right)\right\}$, we have that $u\left(t_{n}, x+x\left(t_{n}\right)\right)$ converges into $L_{x}^{2}\left(\mathbb{R}^{2}\right)$. By Ascoli-Arzela Theorem, we have the following.

Proposition 18. Suppose that the critical mass $m_{0}$ is finite. Then there exists a global solution $u$ with mass $m_{0}$, and for every $\eta>0$ there exists $0<C(\eta)<\infty$ such that

$$
\int_{|x-x(t)| \geq C(\eta)}|u(t, x)|^{2} d x+\int_{|\xi-\xi(t)| \geq C(\eta)}|\widehat{u}(t, \xi)|^{2} d \xi \leq \eta
$$

for all $t \in \mathbb{R}$, where the functions $x, \xi: \mathbb{R} \rightarrow \mathbb{R}^{2}$.

Proposition 19. The solution described in Proposition 18 does not exist.

Once we proved Proposition 19, we can say that $m_{0}=\infty$ and thus Theorem 3 is true. In order to prove Proposition 19, we need the following two lemmas.

Lemma 20 (see [6, Lemma 5.2]). Let $u$ be a global solution of (5). Then one has

$$
\iint_{\mathbb{R} \times \mathbb{R}^{2}} \frac{\langle t\rangle^{2} G(u)}{\langle t\rangle^{3}+|x|^{3}} d x d t \leq C(E),
$$

where $\langle t\rangle=\sqrt{1+|t|^{2}}$.

Lemma 21 (see [6, Lemma 6.2]). Let $u$ be a global solution of (5). Let $B$ be a compact subset of $\mathbb{R}^{2}$. Then for any $R>0$ and $T>0$, one has

$$
\int_{B(R)}|u(T, x)|^{2} d x \geq \int_{B}|u(0, x)|^{2} d x-\frac{C(E) T}{R},
$$

where $B(R):=\left\{x \in \mathbb{R}^{2} \mid \exists y \in B\right.$ s.t. $\left.|x-y|<R\right\}$.

Proof of Proposition 19. By Lemma 21, choosing $\eta$ sufficiently small,

$$
\begin{aligned}
& \int_{|x-x(0)| \leq C(\eta)+R|t|}|u(t, x)|^{2} d x \\
& \geq \int_{|x-x(0)| \leq C(\eta)}|u(0, x)|^{2} d x-\frac{C(E)}{R} \\
& \quad \geq m_{0}-\eta-\frac{C(E)}{R} .
\end{aligned}
$$


By Proposition 18,

$$
\int_{|x-x(t)| \leq C(\eta)}|u(t, x)|^{2} d x \geq m_{0}-\eta .
$$

For a fixed large number $R$, we must have $|x(t)-x(0)| \leq$ $2 C(\eta)+R|t|$. By Lemma 20 and Hölder inequality,

$$
\begin{aligned}
\infty & >\iint_{\mathbb{R} \times \mathbb{R}^{2}} \frac{\langle t\rangle^{2}|u|^{4}}{\langle t\rangle^{3}+|x|^{3}} d x d t \\
& \gtrsim \int_{\mathbb{R}} \int_{|x-x(t)| \leq C(\eta)} \frac{\langle t\rangle^{2}|u|^{4}}{\langle t\rangle^{3}+|x|^{3}} d x d t \\
& \gtrsim \int_{\mathbb{R}} \int_{|x-x(t)| \leq C(\eta)} \frac{|u|^{4}}{\langle t\rangle+1} d x d t \\
& \gtrsim \int_{\mathbb{R}} \int_{|x-x(t)| \leq C(\eta)} \frac{|u|^{2}}{\langle t\rangle+1} d x d t \\
& \geq\left(m_{0}-\eta\right) \int_{\mathbb{R}} \frac{1}{\langle t\rangle+1} d x d t=\infty .
\end{aligned}
$$

This is a contradiction. Proposition 19 was obtained.

\section{Acknowledgment}

This work is supported by China Scholarship Council and NNSF of China (no. 11271023).

\section{References}

[1] M. Nakamura and T. Ozawa, "Nonlinear Schrödinger equations in the Sobolev space of critical order," Journal of Functional Analysis, vol. 155, no. 2, pp. 364-380, 1998.

[2] B. X. Wang, "The smoothness of scattering operators for sinhGordon and nonlinear Schrödinger equations," Acta Mathematica Sinica, vol. 18, no. 3, pp. 549-564, 2002.

[3] B. Wang, C. Hao, and H. Hudzik, "Energy scattering theory for the nonlinear Schrödinger equations with exponential growth in lower spatial dimensions," Journal of Differential Equations, vol. 228, no. 1, pp. 311-338, 2006.

[4] S. Ibrahim, M. Majdoub, N. Masmoudi, and K. Nakanishi, "Scattering for the two-dimensional energy-critical wave equation," Duke Mathematical Journal, vol. 150, no. 2, pp. 287-329, 2009.

[5] J. Colliander, S. Ibrahim, M. Majdoub, and N. Masmoudi, "Energy critical NLS in two space dimensions," Journal of Hyperbolic Differential Equations, vol. 6, no. 3, pp. 549-575, 2009.

[6] K. Nakanishi, "Energy scattering for nonlinear Klein-Gordon and Schrödinger equations in spatial dimensions 1 and 2," Journal of Functional Analysis, vol. 169, no. 1, pp. 201-225, 1999.

[7] R. Killip, T. Tao, and M. Visan, "The cubic nonlinear Schrödinger equation in two dimensions with radial data," Journal of the European Mathematical Society, vol. 11, no. 6, pp. 1203-1258, 2009.

[8] B. Dodson, "Global well-posedness and scattering for the defocusing, $L^{2}$-critical, nonlinear Schrödinger equation when $d=2$, http://128.84.158.119/abs/1006.1375vl.
[9] C. E. Kenig and F. Merle, "Global well-posedness, scattering and blow-up for the energy-critical, focusing, non-linear Schrödinger equation in the radial case," Inventiones Mathematicae, vol. 166, no. 3, pp. 645-675, 2006.

[10] S. Adachi and K. Tanaka, "Trudinger type inequalities in $\mathbb{R}^{N}$ and their best exponents," Proceedings of the American Mathematical Society, vol. 128, no. 7, pp. 2051-2057, 2000.

[11] J. Ginibre and G. Velo, "Scattering theory in the energy space for a class of nonlinear Schrödinger equations," Journal de Mathématiques Pures et Appliquées, vol. 64, no. 4, pp. 363-401, 1985.

[12] J. Bourgain, "Global well-posedness of defocusing 3D critical NLS in the radial case," Journal of American Mathematical Society, vol. 12, pp. 145-171, 1999.

[13] T. Tao, M. Visan, and X. Zhang, "Minimal-mass blowup solutions of the mass-critical NLS," Forum Mathematicum, vol. 20, no. 5, pp. 881-919, 2008.

[14] F. Merle and L. Vega, "Compactness at blow-up time for $L^{2}$ solutions of the critical nonlinear Schrödinger equation in $2 D$," International Mathematics Research Notices, no. 8, pp. 399-425, 1998.

[15] H. Bahouri and P. Gérard, "High frequency approximation of solutions to critical nonlinear wave equations," American Journal of Mathematics, vol. 121, no. 1, pp. 131-175, 1999.

[16] P. Bégout and A. Vargas, "Mass concentration phenomena for the $L^{2}$-critical nonlinear Schrödinger equation," Transactions of the American Mathematical Society, vol. 359, no. 11, pp. 5257$5282,2007$. 


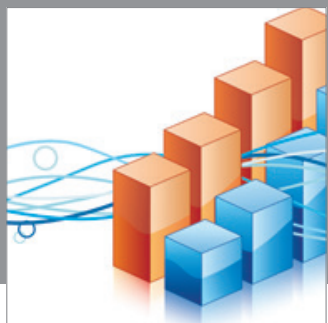

Advances in

Operations Research

mansans

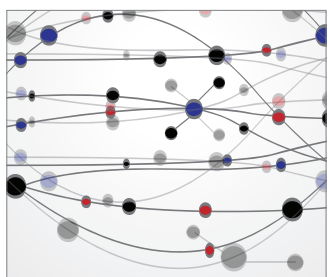

The Scientific World Journal
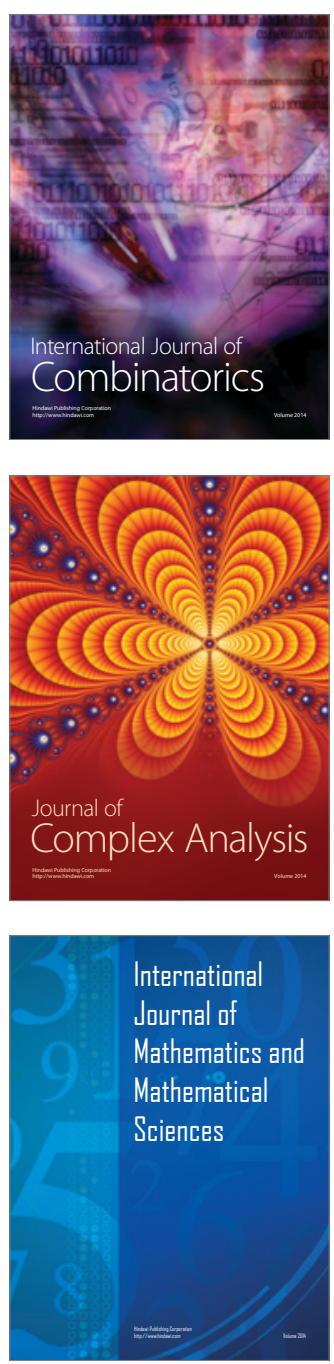
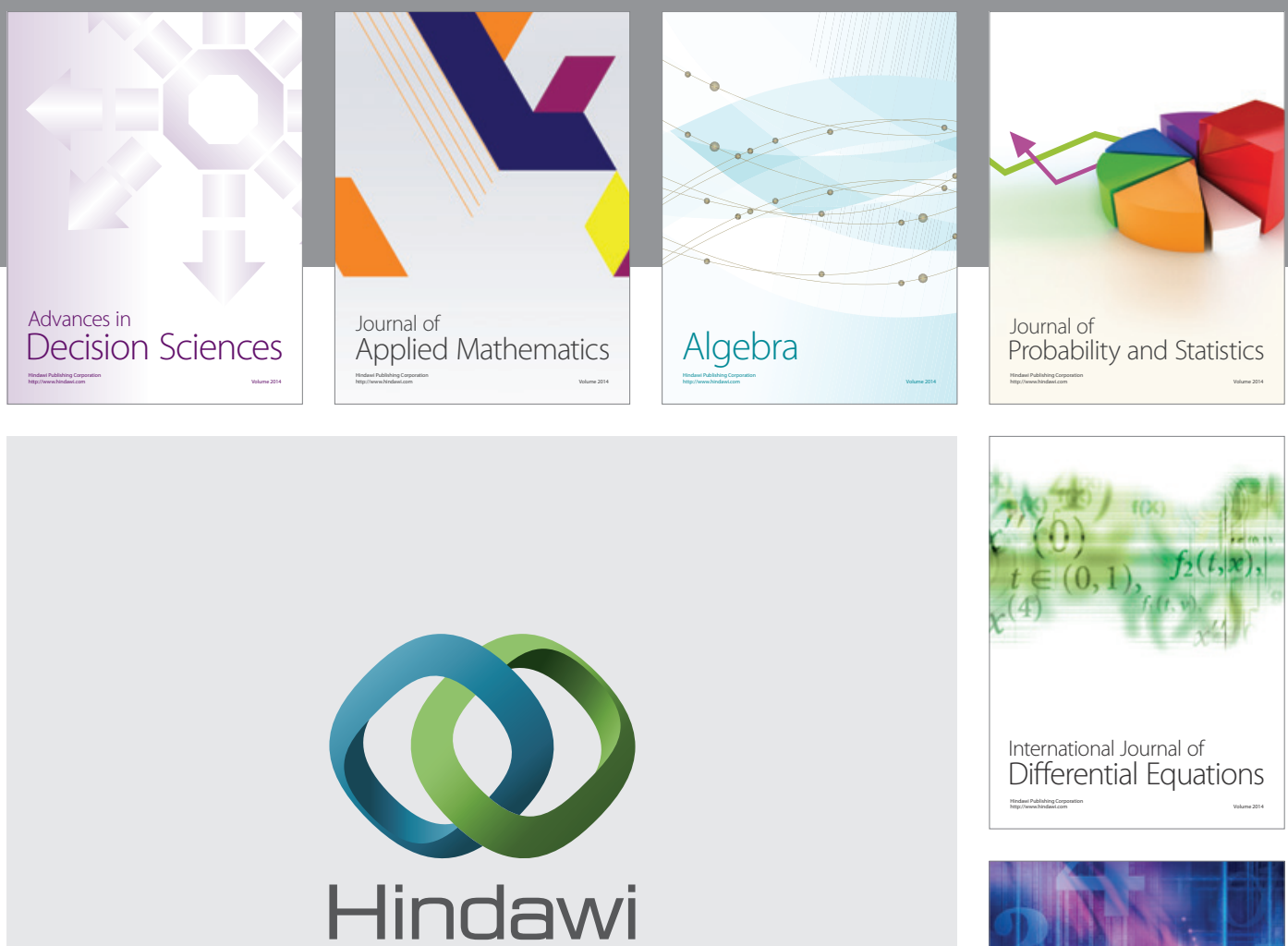

Submit your manuscripts at http://www.hindawi.com
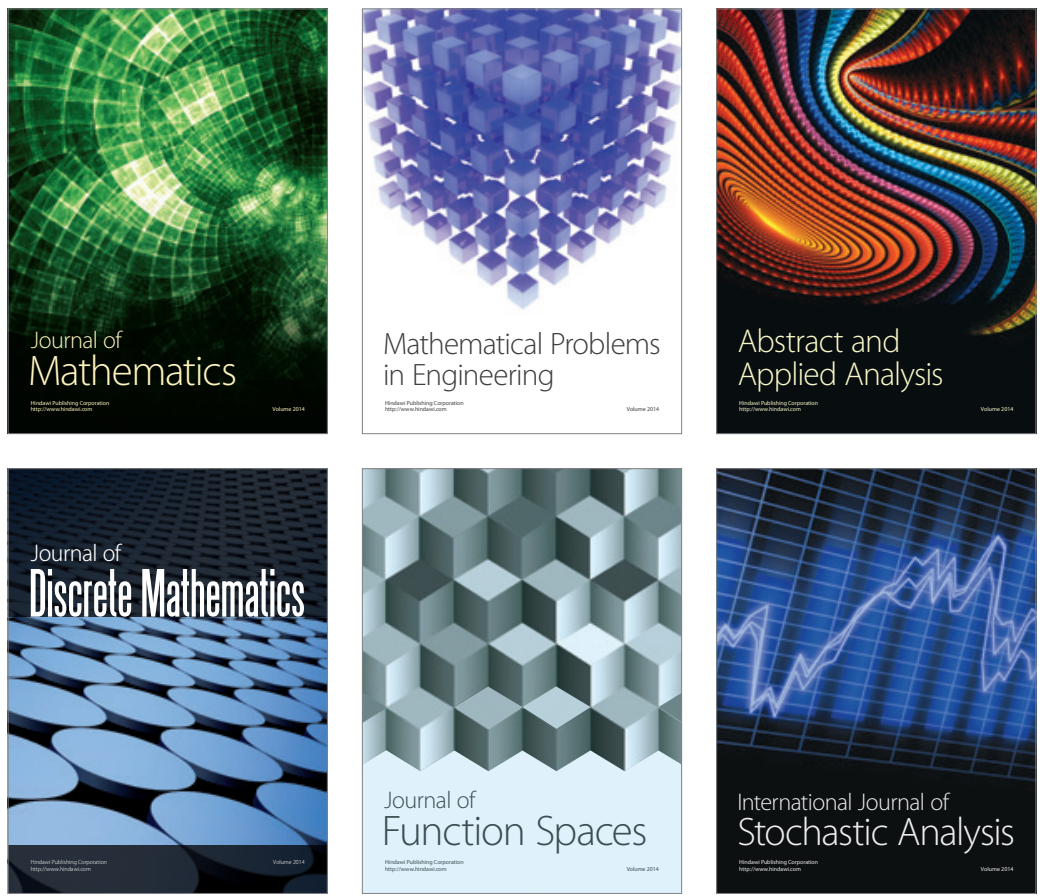

Journal of

Function Spaces

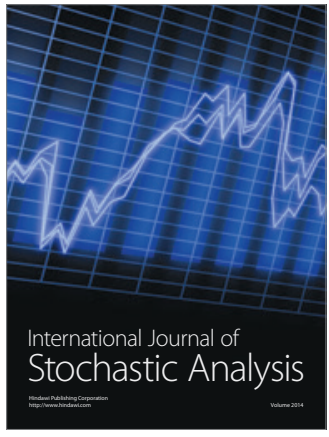

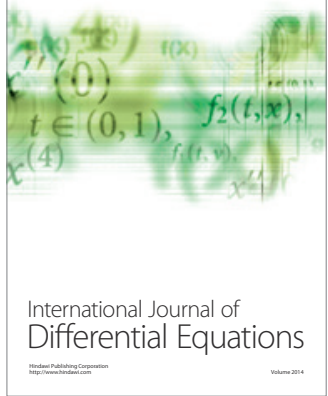
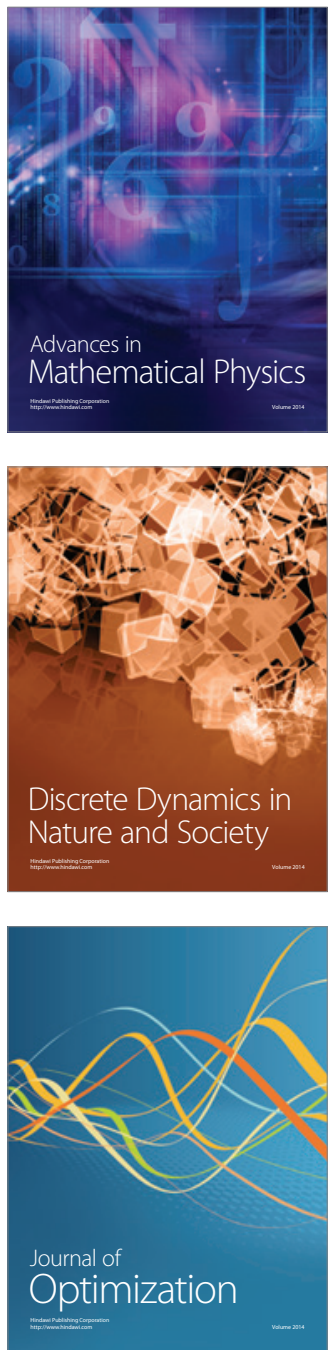